\title{
Cooperative Lossy Communications in Unmanned Aerial Vehicle Networks: Age-of-Information with Outage Probability
}

\author{
Wensheng Lin, Member, IEEE, Lixin Li, Member, IEEE, Jinhong Yuan, Fellow, IEEE, \\ Zhu Han, Fellow, IEEE, Markku Juntti, Fellow, IEEE, and Tad Matsumoto, Life Fellow, IEEE
}

\begin{abstract}
This paper analyzes the robustness and timeliness for cooperative lossy communications in unmanned aerial vehicle (UAV) networks. The analytical framework consists of two steps: 1) calculating the outage probability, and 2) characterizing the Age-of-Information (AoI) for a given outage probability. Initially, we determine the outage probability based on the Shannon's lossy source-channel separation theorem. Numerical results indicate that joint decoding reduces the outage probability, and the system can achieve higher diversity order for less stringent distortion requirement. Then, we derive a closed-form expression of the lower bound on the average AoI for the communication system where outage events are constrained to an acceptable level. Moreover, we conduct a series of simulations for verifying the lower bound on the average AoI and evaluating the impact of UAV locations on the average AoI. It is demonstrated that the lower bound is tight when the server utilization ratio is either relatively busy or idle. For the case with a relatively large outage probability, we propose an intermittent transmission scheme to refine the AoI performance without extra energy consumption. The effectiveness of the intermittent transmission scheme for reducing the average AoI is verified by both the simulations and the lower bound analysis.
\end{abstract}

Index Terms-Unmanned aerial vehicles, outage probability, Age-of-Information, cooperative communications, lossy communications.

\section{INTRODUCTION}

It is universally acknowledged that unmanned aerial vehicles (UAVs) have become an important part of Internet-of-Things (IoT) infrastructure for supporting smart society [1]. In IoT

This work was supported in part by National Natural Science Foundation of China (NSFC) under Grant 62001387, in part by Shanghai Academy of Spaceflight Technology (SAST) under Grant SAST2020124, in part by the Fundamental Research Funds for the Central Universities, in part by NSF CNS-2107216 and EARS-1839818, in part by Toyota, in part by the Australian Research Council (ARC) Discovery Projects under Grant DP190101363, in part by the ARC Linkage Project under Grant LP170101196, and in part by the Academy of Finland 6Genesis Flagship (Grant 318927).

Corresponding author: Wensheng Lin.

W. Lin and L. Li are with the School of Electronics and Information, Northwestern Polytechnical University, Xi'an, Shaanxi 710129, China (e-mail: linwest@nwpu.edu.cn; lilixin@nwpu.edu.cn).

J. Yuan is with the School of Electrical Engineering and Telecommunications, University of New South Wales, Sydney, NSW 2052, Australia (e-mail: j.yuan@unsw.edu.au).

Z. Han is with the Department of Electrical and Computer Engineering in the University of Houston, Houston, TX 77004 USA, and also with the Department of Computer Science and Engineering, Kyung Hee University, Seoul, South Korea, 446-701.

M. Juntti is with the Centre for Wireless Communications, University of Oulu, 90014 Oulu, Finland (e-mail: markku.juntti@oulu.fi).

T. Matsumoto is with the School of Information Science, Japan Advanced Institute of Science and Technology, Ishikawa, Japan 923-1292, Japan, and also with the Centre for Wireless Communications, University of Oulu, 90014 Oulu, Finland (The position in University of Oulu has been frozen since January, 2018) (e-mail: matumoto@jaist.ac.jp).

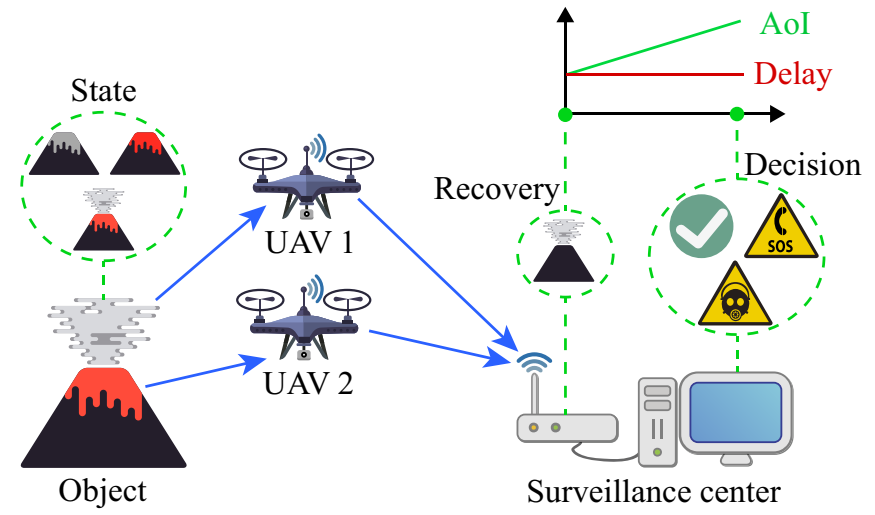

Fig. 1. A UAV cooperative surveillance system for IoT applications.

systems, UAVs can play versatile roles, such as providing multiple access services [2]-[4], replenishing energy of IoT devices [5], [6], and supporting computation offloading [7]. When adopting UAVs in surveillance systems, compared to the devices deployed at a solid location, UAVs can carry cameras and sensors to collect richer information from various altitudes and closer to the object over a rough terrain. For example, Fig. 1 illustrates a typical implementation of UAVs in cooperative operations. A surveillance center wants to monitor the state of a remote object. Therefore, it relies on UAVs to detect the object state conveniently and economically. To obtain more information of the object and increase the robustness of the surveillance system, multiple UAVs are deployed in different locations for cooperative surveillance. Noticed that the information collected by multiple UAVs is correlated for the same object. Hence, the surveillance center can enhance the system performance by cooperative communications, i.e., jointly utilizing the information uploaded from multiple UAVs.

However, there is an inevitable problem that vehicular communications are not always reliable. Specifically, the surveillance center may fail to reconstruct the object information, if the UAVs transmit information under harsh channel conditions. Fortunately, the final goal of IoT is to make further decisions rather than recover the information itself. As long as the decision is correct, the information is not necessarily to be losslessly recovered. Thus, the lossy recovery is still acceptable if its distortion is no larger than a specified distortion requirement. Based on the above reality, cooperative lossy communications in UAV networks are promising for IoT applications.

In some cases, the IoT system may need to perform operations according to the object state. Intuitively, the appropriate- 
ness of an operation is affected by the information freshness of the object state. Conventional performance metrics for timeliness, i.e., delay and latency, are static for each packet. Nonetheless, the information freshness of the object state changes at different time point when the system performs an operation. Therefore, we need a dynamic metric to indicate the timeliness as time elapses. Age-of-Information (AoI) [8] is such a performance metric that continuously characterizes the dynamics of freshness of the object information. It has been utilized in diverse communications and control systems, especially for systems with stringent real-time requirements. AoI is also becoming popular in various applications including scheduling design [9], [10], updating policy design for energy harvesting devices [11], [12], and trade-off between sensing and communication [13]. Hence, we select AoI as the performance metric to evaluate the timeliness of the system. Moreover, since AoI is time-variant, the average AoI can better reflect the system performance during a long time period. Consequently, this paper aims at analyzing the average AoI for cooperative lossy communications in UAV networks.

Definitely, AoI decreases as the surveillance center refresh the state information of the object. As stated above, in IoToriented cooperative lossy communications, the system decision is reliable if the information recoveries in all links satisfy the distortion requirements. Conversely, an outage event occurs once the distortion requirements cannot be all satisfied. In this case, the surveillance center fails to refresh the state information of the object, resulting in AoI keeping increasing. Thus, to analyze AoI for cooperative lossy communications in UAV networks, we need to investigate the robustness of the UAV communications by evaluating the outage probability for the first step.

In the literature, we can find a lot of work related to the calculation of the outage probability for cooperative communication systems. Laneman et al. [14] derived the outage probability for Rayleigh fading in a relaying system applying diverse forwarding strategies. For lossy-forward (LF) relaying systems [15], the outage probabilities over Rayleigh fading and Nakagami- $m$ fading were characterized by Zhou et al. [16] and Qian et al. [17], respectively. Then, Lin et al. [18] extended the results to the LF relaying systems with lossy information reconstructed at the destination. Besides, Qian et al. compared the LF strategy with the decode-and-forward (DF) and the adaptive DF strategies for block Rayleigh fading in [19]. With more than one source in the system, Lu et al. [20] analyzed the outage probability of the orthogonal multiple-access relaying systems suffering from block Rayleigh fading, provided that intra-link errors occurred in the source-relay transmissions. Lin et al. [21] conducted the outage probability analysis for lossy communications with two sources and one helper over block Rayleigh fading channels.

It is noticed that the results in the literature are based on conventional fading models, e.g., original Rayleigh fading and Nakagami- $m$ fading. However, the original Rayleigh and Nakagami- $m$ fading models are too simple for UAV communications, and hence neither of the original fading models is sufficiently accurate to describe the UAV channel conditions. To analyze the performance of the cooperative lossy communications in UAV networks, we need to conduct derivations over UAV channels. As reported in [22], there are large quantities of measurement campaigns for UAV channel modeling. Consequently, we will select the appropriate UAV channel model to calculate the outage probability in UAV cooperative lossy communication systems, and further analyze the AoI performance.

In the UAV cooperative surveillance system considered in this paper, the update of the object state starts from the object changing its state to the surveillance center recovering the object information. Certainly, the start of the update is at one time point, and so is the end of the update due to the joint decoding scheme in the surveillance center. Even though there are two links for information transmission in the system, there is only one update. Hence, from the view of the whole system, the update process can be equivalently regarded as a queueing process with only one queue and one server. If a new update arrives when the whole system is busy, it has to wait until the system finishes processing the previous update. We further assume that the change of the object state follows a Poisson process, while the service time of the whole system follows the exponential distribution. Consequently, this system is equivalent to an $\mathrm{M} / \mathrm{M} / 1$ system with the first-come-firstserved (FCFS) scheme, i.e., a typical queueing model. Here, $\mathrm{M} / \mathrm{M} /$ indicates Poison arrival and exponential service time distributions.

Generally, the queueing model described by " $\mathrm{A} / \mathrm{S} / N_{S} / N_{B}$ " is interpreted as follows. "A" and " $\mathrm{S}$ " represent the arrival and service processes. The symbol "M" specifies the process with Poisson arrivals or exponential service times, and the symbol "D" denotes the process with a deterministic distribution. $N_{S}$ stands for the number of servers. $N_{B}$ is the buffer size of the queue. If not specified, $N_{B}$ is assumed to be infinite.

To date, there are already numerous research achievements with respect to AoI. Early in 2011, Kaul et al. [23] introduced the concept of AoI to optimize the freshness of state information in a vehicular network adopting the IEEE 802.11 protocol. Then, Kaul et al. presented the fundamental characterizations with regard to AoI in [24], [25]. They determined the average AoI for $\mathrm{M} / \mathrm{M} / 1, \mathrm{M} / \mathrm{D} / 1$, and $\mathrm{D} / \mathrm{M} / 1$ systems with the FCFS scheme in [24]. Subsequently, in [25], the average AoI of M/M/1 systems using the last-come-first-served (LCFS) scheme was investigated for the cases that a new arrived packet can or cannot pre-empt the currently served packet. For the AoI analysis in UAV networks, Han et al. [26] calculated the average AoI for successfully transmitted packets in UAVaided IoT networks, which was modeled as the M/M/1 system with the FCFS scheme. Ahani et al. [27] designed a route scheduling algorithm for a UAV with limited battery capacity to minimize the average overall AoI. In [28], Abd-Elmagid and Dhillon minimized the average peak AoI in an IoT network with a UAV relay.

Note that the work shown above implicitly assumes that the communication channels are all reliable in the system. Indubitably, vehicular communications are not always reliable, and the occurrence of an outage event will affect the AoI because a renewal of information is missed. Hence, the above work is not suitable for the performance analysis of 
cooperative lossy communications in UAV networks. In the literature, there is some research work taking packet errors into consideration for AoI analysis as exemplified below. Chen and Huang [29] derived the peak AoI for the M/M/1 system with the FCFS or LCFS scheme. Zhang et al. [30] considered AoI with a successful sensing probability for optimizing sensing and transmission time in a cellular Internet of UAVs. Gu et al. [31] characterized both the average AoI and the average peak AoI for the IoT monitoring system with truncated automatic repeat request scheme. $\mathrm{Gu}$ et al. [32] also analyzed the average peak AoI of both the primary IoT system and the secondary IoT system in an overlay or underlay scheme. Nevertheless, identifying the average AoI for the $M / M / 1$ system with the FCFS scheme under the constraint of certain packet errors has not been finished yet.

In brief, the analytical framework of cooperative lossy communications in UAV networks consists of the following two steps: 1) calculating the outage probability for the system, and 2) determining the average AoI for a given outage probability. The contributions of this paper are summarized as follows:

- We establish the analytical framework for the outage probability and the average AoI of cooperative lossy communications in UAV networks. When calculating the outage probability, it is difficult to directly determine the signal-to-noise ratio (SNR) thresholds for satisfying distortion requirements in cooperative communications. We solve this problem by formulating an equivalent multiterminal source coding problem based on the Shannon's lossy source-channel separation theorem [33], [34].

- We derive a lower bound on the average AoI for the M/M/1 system with the FCFS scheme and accepting outage events. Specifically, we start from two extreme cases, i.e., the idle and busy cases, and then combine the results from the two cases together to obtain a relatively tight lower bound on the average AoI.

- We conduct a series of simulations to compare the lower bound with the exact AoI. The curves of the lower bound and the simulation results have very small differences when the system is either relatively busy or idle.

- To refine the AoI performance, we propose an intermittent transmission scheme to reduce the average AoI when the outage probability is relatively large. The principle is to allocate more energy to reduce the outage probability rather than increase the updating frequency, if the relatively large outage probability becomes the dominant factor of AoI. Both the simulation results and the lower bound verify that intermittent transmission can reduce the average AoI without additional energy consumption.

- We evaluate the impact on AoI due to different UAV locations based on a practical scenario. The results show that the average AoI keeps at a low level if the UAVs are located within a specific range, while it rapidly increases if the UAVs move outside of the specific range.

The rest of this paper is organized as follows. Section II describes the system model and the channel model of the UAV cooperative surveillance system in detail. Section III analyzes the outage probability, and Section IV further derives a lower bound of the average AoI. The lower bound is compared with simulation results in Section V. Finally, we conclude this work in Section VI.

Throughout this paper, we use the standard notation rules as follows. The random variables and their realizations are denoted by uppercase and lowercase letters, respectively. Calligraphic letters $\mathcal{X}$ and $\mathcal{R}$ denote the corresponding finite sets. The base of $\operatorname{logarithm}$ function $\log (\cdot)$ is assumed to be 2 unless specified.

\section{Problem Statement}

We present first the details of the communication model for the UAV cooperative surveillance system in Section II-A. Then, Section II-B and Section II-C introduce the corresponding multiterminal source coding problem and the channel model, respectively, for deriving the outage probability.

\section{A. System Model}

As depicted in Fig. 1, the simplest scenario of the UAV cooperative surveillance system consists of one object, two UAVs and one surveillance center. The UAVs are deployed in different locations to monitor the same object, whose state changes randomly. Once the UAVs detect the change of the object state, they separately collect the object information and upload it to the surveillance center. Since the UAVs monitor the same object, correlation exists in the object information collected by the UAVs. Therefore, the surveillance center is able to minimize the distortion of the object information by jointly decoding after receiving the signals from multiple UAVs.

However, when suffering from the channel fading, the transmissions in UAV communication systems are not always reliable. Therefore, the recoveries of the object information may contain distortions if the channel condition is not sufficiently good. Notice that the goal of the IoT system is not only to recover the object information, but also to make some decisions and consequent operations. It means that the distortions are acceptable as long as the surveillance center can still make correct inference. The maximum acceptable distortions are given by the distortion requirements $\left(D_{1}, D_{2}\right)$ for two links. Once either of the distortion requirements cannot be satisfied, the recoveries are discarded and an outage event occurs. In this case, the surveillance center has to utilize the previous acceptable recoveries for making further decisions. Certainly, the decision may be not sufficiently appropriate due to the reduction of the information freshness for the object state.

For the purpose of dynamically evaluating the information freshness in the system, AoI is introduced and defined as the elapsed time of the latest served update from the generation of the update. In the UAV cooperative lossy communication system, AoI fails in updating and keeps increasing if an outage event occurs. In order to analyze AoI of the system illustrated in Fig. 1, we need to evaluate the outage probability for the first step, and then derive the AoI for a specified value of the outage probability. 


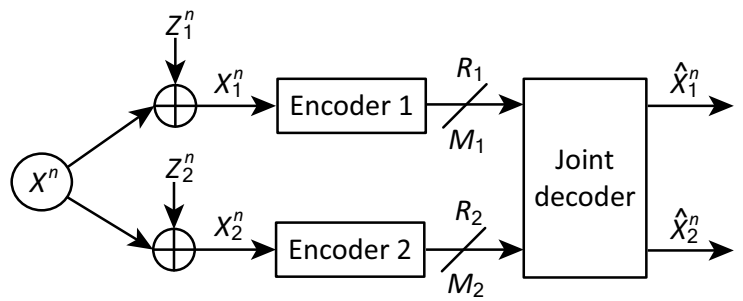

Fig. 2. The multiterminal source coding problem for outage probability analysis.

The conventional way to calculate the outage probability is to determine the instantaneous SNR of the received signals for given channel gains. Subsequently, the outage probability is calculated by the integral with respect to the instantaneous SNR distribution that cannot achieve the threshold for successful decoding. However, in cooperative communication systems with correlated sources, it is not easy to directly determine the SNR thresholds according to specified distortion requirements.

To solve the problem of determining the SNR thresholds, we utilize a two-step method to equivalently derive the relationship between the instantaneous SNR and the final distortions. Initially, given an instantaneous SNR, we can calculate the corresponding achievable rate. Then, the transmitter compresses the information at the code rate satisfying the instantaneous achievable rate. The distortions can be evaluated by solving a multiterminal source coding problem. Based on the Shannon's lossy source-channel separation theorem, we will finally establish the relationship between the instantaneous SNRs and the distortions. Conversely, given the distortion requirements, we will determine the achievable link rates and further obtain the SNR thresholds.

\section{B. Multiterminal Source Coding Problem}

Fig. 2 illustrates the multiterminal source coding problem corresponding to the UAV cooperative surveillance system. There is a common source $X^{n}$ separately observed by two encoders while suffering from random noise processes $Z_{1}^{n}$ and $Z_{2}^{n}$, where $n$ denotes the sequence length. In practical systems, it is reasonable to assume that the source is Gaussian. Consequently, the observations $X_{1}^{n}$ and $X_{2}^{n}$ can be regarded as two correlated Gaussian sources ${ }^{1}$. Then, two distributed encoders compress the observations $X_{1}^{n}$ and $X_{2}^{n}$ into two codewords $M_{1}$ and $M_{2}$ at the code rates $R_{1}$ and $R_{2}$, respectively. Since the source is Gaussian, the encoder quantizes the observation and then maps it into a codeword taking values in a finite alphabet. Therefore, the code rates can be larger than 1 . After receiving two codewords $M_{1}$ and $M_{2}$, the joint decoder recovers the estimates of two observations as $\hat{X}_{1}^{n}$ and $\hat{X}_{2}^{n}$ by exploiting the correlations between the observations. Constrained by the link rates, the recovered signals $\hat{X}_{1}^{n}$ and $\hat{X}_{2}^{n}$ may not be lossless, and hence, the distortion measure $d_{i}: \mathcal{X}_{i} \times \hat{\mathcal{X}}_{i} \mapsto[0, \infty)$ for $i \in\{1,2\}$ is defined to describe the distortion degree between two symbols

\footnotetext{
${ }^{1}$ In essence, the system is a special case of communications with correlated sources, and hence we can design the encoder and the joint decoder for practical implemetations according to [35].
}

$x_{i}$ and $\hat{x}_{i}$. Furthermore, the average distortion of the whole sequence is defined as

$$
d_{i}\left(x_{i}^{n}, \hat{x}_{i}^{n}\right)=\frac{1}{n} \sum_{t=1}^{n} d_{i}\left(x_{i}(t), \hat{x}_{i}(t)\right) .
$$

In general, the distortion is evaluated by quadratic (squared error) distortion measure $d_{\mathrm{Q}, i}\left(x_{i}, \hat{x}_{i}\right)=\left(x_{i}-\hat{x}_{i}\right)^{2}$ for Gaussian sources. Given the distortion requirements $\left(D_{1}, D_{2}\right)$, the achievable rate-distortion region $\mathcal{R}\left(D_{1}, D_{2}\right)$, which consists of all achievable rate pairs $\left(R_{1}, R_{2}\right)$, is defined as

$$
\begin{aligned}
\mathcal{R}\left(D_{1}, D_{2}\right) & \\
=\left\{\left(R_{1}, R_{2}\right):\right. & \left(R_{1}, R_{2}\right) \text { is admissible such that } \\
& \lim _{n \rightarrow \infty} \mathrm{E}\left(d_{i}\left(x_{i}^{n}, \hat{x}_{i}^{n}\right)\right) \leq D_{i}+\epsilon, \\
& \text { for } i=1,2, \text { and any } \epsilon>0\} .
\end{aligned}
$$

The achievable rate-distortion region establishes the connection between the link rates and the distortions.

\section{Channel Model}

In order to characterize the channel conditions and further determine the constraints on link rates, we have to select the appropriate channel models. Since the distortions are defined between $X_{i}^{n}$ and $\hat{X}_{i}^{n}$, we only need to consider the channels from the UAV to the surveillance center, i.e., typical air-to-ground (A2G) channels. We further assume that the A2G channel conditions depend on the elevation angle of the UAV and the impact of environment including line-of-sight (LoS) and non-LoS (NLoS) conditions. According to [36, Eq. (2)], given the transmission power $P_{\mathrm{UAV}, i}$ of the $i$-th $\mathrm{UAV}$, the power of the $i$-th signal received in the surveillance center can be expressed as

$$
P_{\mathrm{SC}, i}=\frac{P_{\mathrm{UAV}, i}|g|^{2}}{P L_{i}},
$$

where $|g|^{2}$ is the fading gain, and $P L_{i}$ stands for the A2G propagation path-loss. The path-loss in $\mathrm{dB}$ is given by [37]

$$
P L_{i}(\mathrm{~dB})=10 \log _{10} P L_{i}=\frac{\eta_{\mathrm{LoS}}-\eta_{\mathrm{NLoS}}}{1+a e^{-b\left(\theta_{i}-a\right)}}+\xi_{i},
$$

where $\eta_{\mathrm{LoS}}, \eta_{\mathrm{NLOS}}, a$ and $b$ are the environmental parameters. $\theta_{i}=\arctan \left(\frac{h_{i}}{s_{i}}\right)$ is the elevation angle, with $h_{i}$ being the altitude of the $i$-th UAV, and $s_{i}$ being the horizontal distance between the $i$-th UAV and the surveillance center. Furthermore,

$$
\xi_{i}=20 \log _{10}\left(\frac{4 \pi f_{i} d_{\mathrm{SC}, i}}{c}\right)+\eta_{\mathrm{NLoS}},
$$

where $d_{\mathrm{SC}, i}=\sqrt{s_{i}^{2}+h_{i}^{2}}$ is the distance between the $i$-th UAV and the surveillance center. $f_{i}$ is the carrier frequency for $i$-th $\mathrm{UAV}$, and $c$ is the speed of light.

We assume that two UAVs utilize different carrier frequency for communications. Let $N_{0}$ be the noise power spectral density of the thermal additive Gaussian noise in the receiver. Then, the instantaneous SNR in each link can be calculated by

$$
\gamma_{i}=\frac{P_{\mathrm{SC}, i}}{N_{0}}=\frac{P_{\mathrm{UAV}, i}|g|^{2}}{N_{0} \cdot P L_{i}} .
$$


Hereafter, we consider the average fading power normalized to unity or $\mathrm{E}\left(|g|^{2}\right)=1$. Thereby, the average SNR in each link is

$$
\bar{\gamma}_{i}=\frac{P_{\mathrm{UAV}, i}}{N_{0} \cdot P L_{i}},
$$

and we have $|g|^{2}=\frac{\gamma_{i}}{\bar{\gamma}}$ for the instantaneous channel power gain. As summarized in [22], many measurement campaigns demonstrate that the Nakagami- $m$ model characterizes the UAV fading channel well. Consequently, we apply the Nakagami- $m$ model to $|g|^{2}$, and, hence, the probability density function (PDF) of $|g|^{2}$ follows the Gamma distribution. Then, it is easy to derive the PDF of $\gamma_{i}$ as

$$
p\left(\gamma_{i}\right)=\frac{m^{m} \gamma_{i}^{m-1}}{\bar{\gamma}_{i}^{m} \Gamma(m)} \exp \left(-\frac{m \gamma_{i}}{\bar{\gamma}_{i}}\right)
$$

where $\Gamma(\cdot)$ represents the Gamma function [38, Eq. (4-35)].

\section{Outage Probability Analysis}

To begin with, Section III-A analyzes the condition for the occurrence of an outage event, and then presents the derivation results of the outage probability. The numerical results of the outage probability are further discussed in Section III-B.

\section{A. Outage Probability Derivation}

Consider two correlated Gaussian sources with covariance matrix

$$
\Lambda=\left(\begin{array}{cc}
\sigma_{1}^{2} & q \sigma_{1} \sigma_{2} \\
q \sigma_{1} \sigma_{2} & \sigma_{2}^{2}
\end{array}\right),
$$

where $\sigma_{i}^{2}$ is the variance of the $i$-th Gaussian source, and $q$ represents the correlation degree of two sources. The achievable rate-distortion region is the set of rate pairs $\left(R_{1}, R_{2}\right)$ such that [39]

$$
\begin{aligned}
R_{1} & \geq \varphi_{1}\left(R_{2}\right), \\
R_{2} & \geq \varphi_{2}\left(R_{1}\right), \\
R_{1}+R_{2} & \geq \psi,
\end{aligned}
$$

where

$$
\begin{aligned}
\varphi_{i}\left(R_{j}\right) & =\frac{1}{2} \log ^{+}\left[\frac{\sigma_{i}^{2}}{D_{i}}\left(1-q^{2}+q^{2} 2^{-2 R_{j}}\right)\right], \\
\psi & =\frac{1}{2} \log ^{+}\left[\left(1-q^{2}\right) \frac{\beta \sigma_{1}^{2} \sigma_{2}^{2}}{2 D_{1} D_{2}}\right],
\end{aligned}
$$

for $i \in\{1,2\}, j=\{1,2\} \backslash i$, with $\log ^{+}(\cdot)=\max \{\log (\cdot), 0\}$ and

$$
\beta=1+\sqrt{1+\frac{4 q^{2} D_{1} D_{2}}{\left(1-q^{2}\right)^{2} \sigma_{1}^{2} \sigma_{2}^{2}}} .
$$

According to the Shannon's lossy source-channel separation theorem, the distortions remain below the given values $\left(D_{1}, D_{2}\right)$ if [40, Theorem 3.7]

$$
R_{i} \leq \Theta_{i}\left(\gamma_{i}\right)=\frac{C\left(\gamma_{i}\right)}{r_{i}}, \text { for } i=1,2,
$$

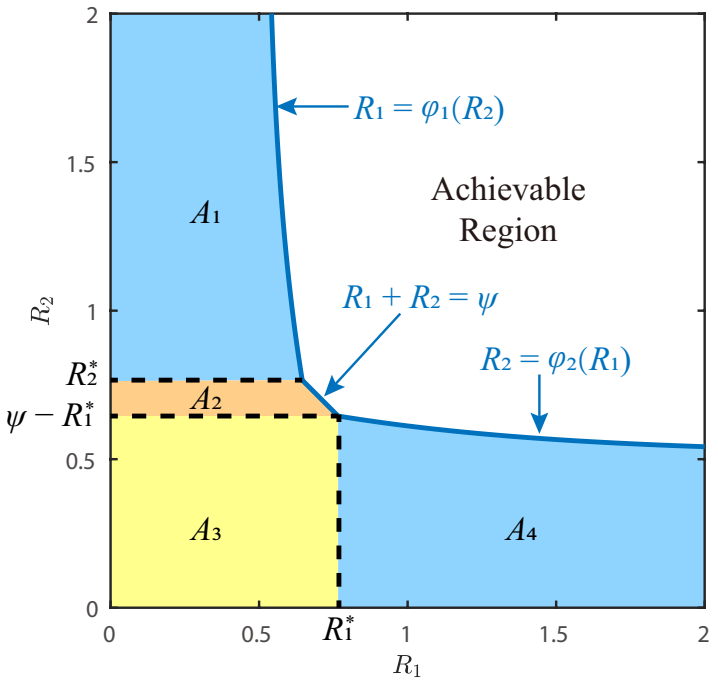

Fig. 3. Achievable rate-distortion region and outage region.

where $C\left(\gamma_{i}\right)$ is the Shannon capacity using the Gaussian codebook, and $r_{i}$ represents the end-to-end rates of joint source-channel coding.

Fig. 3 illustrates the achievable rate-distortion region for two Gaussian sources. In order to clearly display the shape of the achievable rate-distortion region, we set $q=0.6$, $\sigma_{1}=\sigma_{2}=0.8$ and $D_{1}=D_{2}=0.2$ as an example. Obviously, an outage event occurs once the rate pair $\left(R_{1}, R_{2}\right)$ falls outside of the achievable rate-distortion region, and hence the outage region is the area outside of the achievable rate-distortion region. Since the instantaneous channel capacities are random variables, the outage probability is the expected value with respect to the link rates supported by the instantaneous channel capacities. To facilitate the integral calculation of outage probability, the outage region is divided into four sub-regions as:

$$
\begin{aligned}
& A_{1}=\left\{0 \leq R_{1}<\varphi_{1}\left(R_{2}\right), R_{2}^{*} \leq R_{2}\right\}, \\
& A_{2}=\left\{0 \leq R_{1}<\psi-R_{2}, \psi-R_{1}^{*} \leq R_{2}<R_{2}^{*}\right\}, \\
& A_{3}=\left\{0 \leq R_{1}<R_{1}^{*}, 0 \leq R_{2}<\psi-R_{1}^{*}\right\}, \\
& A_{4}=\left\{R_{1}^{*} \leq R_{1}, 0 \leq R_{2}<\varphi_{2}\left(R_{1}\right)\right\},
\end{aligned}
$$

where $\left(R_{1}^{*}, \psi-R_{1}^{*}\right)$ and $\left(\psi-R_{2}^{*}, R_{2}^{*}\right)$ are the corner points to the achievable rate-distortion region, and hence $R_{i}^{*}$ is the solution of $\psi-\varphi_{j}\left(R_{i}\right)-R_{i}=0$. Therefore, the outage probability for the UAV cooperative surveillance system can be calculated by

$$
P_{\mathrm{out}}=\sum_{k=1}^{4} P_{k}
$$

where $P_{k}$ represents the outage probability for sub-region $A_{k}$. The derivation results of $P_{k}$ are

$$
\begin{aligned}
P_{1}= & \frac{\Gamma\left(m, \frac{m}{\bar{\gamma}_{2}} \Theta_{2}^{-1}\left(R_{2}^{*}\right)\right)}{\Gamma(m)}-\frac{m^{m}}{\bar{\gamma}_{2}^{m}[\Gamma(m)]^{2}} \int_{\Theta_{2}^{-1}\left(R_{2}^{*}\right)}^{\infty} \gamma_{2}^{m-1} \\
& \cdot \exp \left(-\frac{m \gamma_{2}}{\bar{\gamma}_{2}}\right) \Gamma\left(m, \frac{m}{\bar{\gamma}_{1}} \Theta_{1}^{-1}\left(\varphi_{1}\right)\right) d \gamma_{2},
\end{aligned}
$$




$$
\begin{aligned}
P_{2}= & \frac{\Gamma\left(m, \frac{m}{\bar{\gamma}_{2}} \Theta_{2}^{-1}\left(\psi-R_{1}^{*}\right)\right)-\Gamma\left(m, \frac{m}{\bar{\gamma}_{2}} \Theta_{2}^{-1}\left(R_{2}^{*}\right)\right)}{\Gamma(m)} \\
& -\frac{m^{m}}{\bar{\gamma}_{2}^{m}[\Gamma(m)]^{2}} \int_{\Theta_{2}^{-1}\left(\psi-R_{1}^{*}\right)}^{\Theta_{2}^{-1}\left(R_{2}^{*}\right)} \gamma_{2}^{m-1} \exp \left(-\frac{m \gamma_{2}}{\bar{\gamma}_{2}}\right) \\
& \cdot \Gamma\left(m, \frac{m}{\bar{\gamma}_{1}} \Theta_{1}^{-1}\left[\psi-\Theta_{2}\left(\gamma_{2}\right)\right]\right) d \gamma_{2} \\
P_{3}= & {\left[1-\frac{\Gamma\left(m, \frac{m}{\bar{\gamma}_{1}} \Theta_{1}^{-1}\left(R_{1}^{*}\right)\right)}{\Gamma(m)}\right] } \\
& \cdot\left[1-\frac{\Gamma\left(m, \frac{m}{\bar{\gamma}_{2}} \Theta_{2}^{-1}\left(\psi-R_{1}^{*}\right)\right)}{\Gamma(m)}\right] \\
P_{4}= & \frac{\Gamma\left(m, \frac{m}{\bar{\gamma}_{1}} \Theta_{1}^{-1}\left(R_{1}^{*}\right)\right)}{\Gamma(m)}-\frac{m}{\bar{\gamma}_{1}^{m}[\Gamma(m)]^{2}} \int_{\Theta_{1}^{-1}\left(R_{1}^{*}\right)}^{\infty} \gamma_{1}^{m-1} \\
& \cdot \exp \left(-\frac{m \gamma_{1}}{\bar{\gamma}_{1}}\right) \Gamma\left(m, \frac{m}{\bar{\gamma}_{2}} \Theta_{2}^{-1}\left(\varphi_{2}\right)\right) d \gamma_{1},
\end{aligned}
$$

where $\Gamma(\cdot, \cdot)$ stands for the upper incomplete gamma function. The detailed derivations of $P_{k}$ are presented in Appendix A. Notice that $\varphi_{1}\left(R_{2}\right)$ defined in (13) is a function of $R_{2}$ and hence also a function of $\gamma_{2}$. Therefore, it is difficult to further derive a closed-form solution from (22). Instead, we will show the numerical results later to discuss the impact on the outage probability due to different parameter settings.

\section{B. Numerical Results}

For the comparison of performance gain between joint decoding and separate decoding, the outage probability of separate decoding is derived as follows.

The rate-distortion function for a Gaussian source $X_{i} \sim$ $N\left(0, \sigma_{i}^{2}\right)$ is [41, Theorem 10.3.2]

$$
R_{i}\left(D_{i}\right)=\frac{1}{2} \log ^{+}\left(\frac{\sigma_{i}^{2}}{D_{i}}\right) .
$$

Thus, the outage event does not occur only when the instantaneous channel capacities are no less than $R_{i}\left(D_{i}\right)$ in both links. Based on the Shannon's lossy source-channel separation theorem, the outage probability of separate decoding can be calculated as

$$
\begin{aligned}
P_{\text {out }}^{\mathrm{S}}= & 1-\int_{\Theta_{1}^{-1}\left[R_{1}\left(D_{1}\right)\right]}^{\infty} p\left(\gamma_{1}\right) d \gamma_{1} \cdot \int_{\Theta_{2}^{-1}\left[R_{2}\left(D_{2}\right)\right]}^{\infty} p\left(\gamma_{2}\right) d \gamma_{2} \\
= & 1-\frac{\Gamma\left(m, \frac{m}{\bar{\gamma}_{1}} \Theta_{1}^{-1}\left[\frac{1}{2} \log ^{+}\left(\frac{\sigma_{1}^{2}}{D_{1}}\right)\right]\right)}{\Gamma(m)} \\
& \cdot \frac{\Gamma\left(m, \frac{m}{\bar{\gamma}_{2}} \Theta_{2}^{-1}\left[\frac{1}{2} \log ^{+}\left(\frac{\sigma_{2}^{2}}{D_{2}}\right)\right]\right)}{\Gamma(m)} .
\end{aligned}
$$

Fig. 4 presents the numerical results of the outage probability for cooperative communications with two correlated Gaussian sources. Clearly, the outage probability is reduced by joint decoding for correlated sources with arbitrary correlation degree. There is no doubt that the outage event occurs less frequently if the distortion requirements become less strict, i.e., $D_{i}$ increases. By comparing Fig. 4(a) and Fig. 4(b), we can

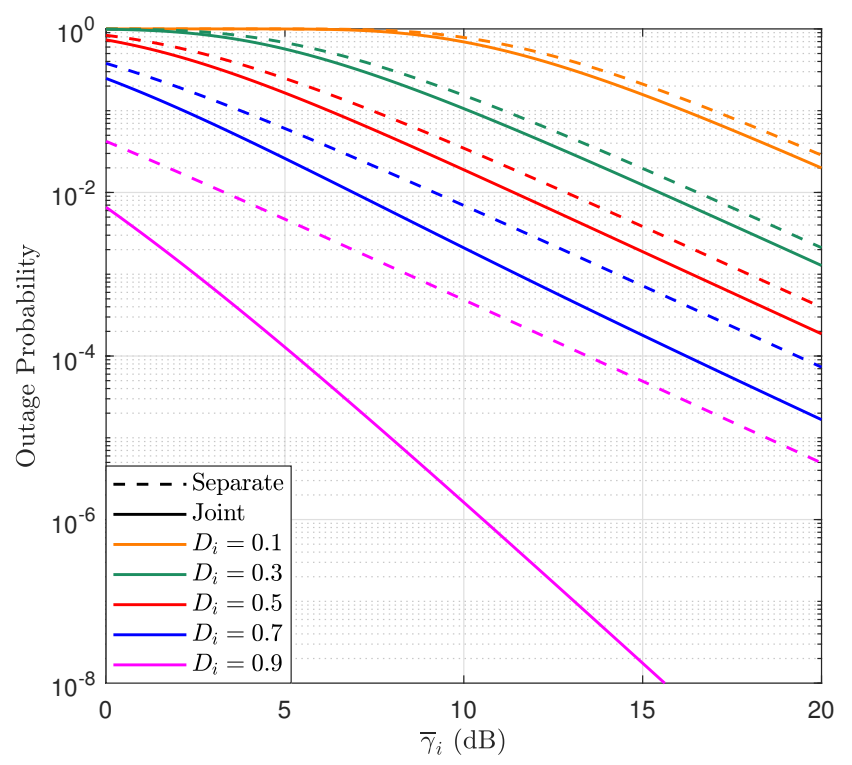

(a) $q=0.4$.

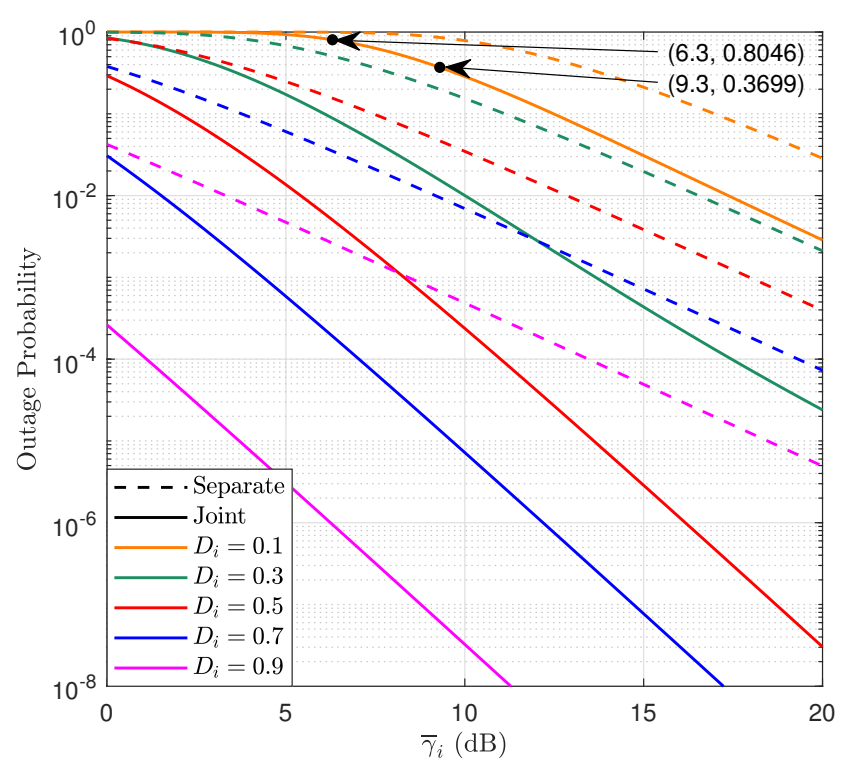

(b) $q=0.8$.

Fig. 4. Outage probability for cooperative communications, where $m=2$, $\sigma_{1}=\sigma_{2}=1$ and $r_{1}=r_{2}=1$.

find that the more correlated the sources are, i.e., $q$ is larger, the lower the outage probability can be. Interestingly, joint decoding also increases the diversity order, which is influenced by both the correlation degree and the distortion requirements. As shown in Fig. 4(a), the slopes of curves obviously change from $D_{i}=0.7$ to 0.9 , and hence, the system with less strict distortion requirements can obtain more diversity gains. However, it is found that the obvious diversity gains appear from $D_{i}=0.1$ to 0.3 and also 0.3 to 0.5 in Fig. 4(b). This observation demonstrates that it is easier to obtain diversity gains with more correlated sources. In addition, we can observe that the performance gains from $D_{i}=0.1$ to 0.3 and from $D_{i}=0.7$ to 0.9 are larger than that from $D_{i}=0.3$ to 0.5 and from $D_{i}=0.5$ to 0.7 . The reason for this observation 


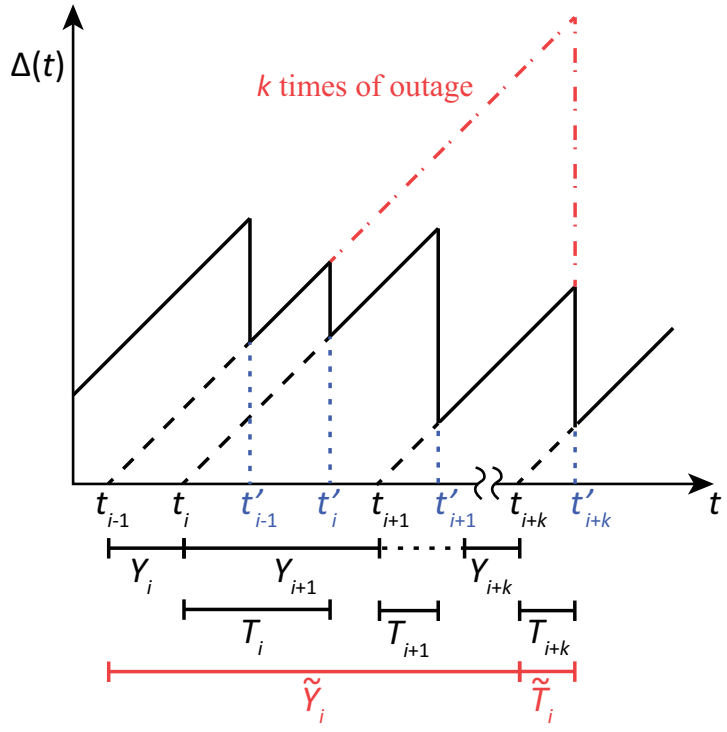

Fig. 5. The AoI when outage events occur.

is explained as follows. The distortion requirement determines the SNR threshold for successful transmissions, and the SNR threshold further determines the outage probability. Since the rate-distortion function is convex, the required rate decreases fast as the distortion requirement becomes less stringent for a small range of $D_{i}$, resulting in a7 significant reduction of the SNR threshold. Hence, the outage probability also decreases fast as a relatively small $D_{i}$ increases. When $D_{i}$ is relatively large, although the SNR threshold becomes very small and does not significantly change, the outage probability reduces fast as the small SNR threshold decreases. Consequently, the performance gain is more obvious when the distortion requirement is relatively small or relatively large.

\section{AoI ANALYSis}

Section IV-A first presents the general analytical framework of the average AoI for a given value of outage probability. Then, in Section IV-B, we consider the average AoI analysis for two extreme cases as follows:

- Busy case: The queue is never empty and the system continuously serves the updates without break.

- Idle case: The queue is always empty and the system must have a break after serving an update.

We find that the results of these two cases provide the lower bounds on the average AoI. Therefore, we finally obtain a relatively tight lower bound by combining the lower bounds for busy and idle cases together.

\section{A. General Analytical Framework}

As stated above, this system can be regarded as an M/M/1 System with the FCFS scheme. Fig. 5 illustrates the AoI in the general case that outage events occur $k$ times continuously. Assume that the $i$-th update is generated at the time $t_{i}$ and the service completed at $t_{i}^{\prime}$. Then, $Y_{i}$ is the interarrival between the $(i-1)$-th and the $i$-th updates, i.e., $Y_{i}=t_{i}-t_{i-1}$. Moreover, $T_{i}$ is the time for the $i$-th update staying at the system, i.e., the system time corresponding to the sum of the queue waiting time $W_{i}$ and service time $S_{i}$. Therefore, we have $T_{i}=t_{i}^{\prime}-t_{i}=$ $W_{i}+S_{i}$, and $W_{i}=\left(T_{i-1}-Y_{i}\right)^{+}$. The update is generated and served at mean rate $\lambda$ and $\mu$, respectively, and hence, the server utilization ratio is $\rho=\frac{\lambda}{\mu}$. The AoI $\Delta(t)$ is the time elapsed from the generation of the last served update, and the average AoI for an interval $(0, \mathcal{T})$ is defined as

$$
\Delta=\frac{1}{\mathcal{T}} \int_{0}^{\mathcal{T}} \Delta(t) d t
$$

where the integral can be calculated by the area under $\Delta(t)$.

In Fig. 5, the outage events occur from the $i$-th update at $t_{i}^{\prime}$ to the $(i+k-1)$-th update at $t_{i+k-1}^{\prime}$, and the $(i+k)$-th update is successful at $t_{i+k}^{\prime}$. Obviously, the AoI keeps increasing if an outage event occurs. Given the outage probability $P_{\text {out }}$, the probability of outage events occurring exactly $k$ times continuously is $P_{\text {out }}^{k}\left(1-P_{\text {out }}\right)$, where $P_{\text {out }}^{k}$ means that the outage events occur at the previous $k$ transmissions and $\left(1-P_{\text {out }}\right)$ means no outage event at the $(k+1)$-th transmission. Let $\Delta_{(k)}$ represent the average AoI for outage events occurring $k$ times continuously. For a sufficiently large number $N_{T}$ of transmissions, the total area under $\Delta(t)$ is

$$
\sum_{k=0}^{\infty} N_{T} P_{\text {out }}^{k}\left(1-P_{\text {out }}\right) \cdot(k+1) \mathrm{E}[Y] \Delta_{(k)}
$$

and the total time is

$$
\sum_{k=0}^{\infty} N_{T} P_{\text {out }}^{k}\left(1-P_{\text {out }}\right) \cdot(k+1) \mathrm{E}[Y] .
$$

Hence, the average AoI can be calculated by the total area under $\Delta(t)$ averaged by the total time, i.e.,

$$
\Delta=\frac{\sum_{k=0}^{\infty} P_{\text {out }}^{k}\left(1-P_{\text {out }}\right)(k+1) \Delta_{(k)}}{\sum_{k=0}^{\infty} P_{\text {out }}^{k}\left(1-P_{\text {out }}\right)(k+1)} .
$$

It is clear that $\Delta_{(0)}$ is the same as the original AoI for completely reliable communications without outage, i.e., [8, Eq. (2.11)-(2.12)]

$$
\begin{aligned}
\Delta_{(0)} & =\frac{\mathrm{E}\left[(Y+T)^{2}\right]-\mathrm{E}\left[T^{2}\right]}{2 \mathrm{E}[Y]} \\
& =\frac{1}{\mu}\left(1+\frac{1}{\rho}+\frac{\rho^{2}}{1-\rho}\right) .
\end{aligned}
$$

For $\Delta_{(k)}$ with general value of $k$, since the $i$-th to the $(i+$ $k-1)$-th updates are lost, we can set two new variables $\tilde{Y}_{i}=$ $\sum_{j=i}^{i+k} Y_{j}$ and $\tilde{T}_{i}=T_{i+k}$. Then, the calculation of AoI follows the same form as $\Delta_{(0)}$, i.e., we have

$$
\Delta_{(k)}=\frac{\mathrm{E}\left[\left(\tilde{Y}_{i}+\tilde{T}_{i}\right)^{2}\right]-\mathrm{E}\left[\tilde{T}_{i}^{2}\right]}{2 \mathrm{E}\left[\tilde{Y}_{i}\right]} .
$$

\section{B. Lower Bounds on AoI}

Since the queue gradually changes from busy to idle as the server utilization ratio $\rho$ decreases from 1 to 0 , the busy and idle cases are two extreme cases for $\rho=1$ and 0 , respectively. Based on the systems in busy and idle cases, we can derive two lower bounds of $\Delta_{(k)}$ as follows. 
Proposition 1 (busy case): $\Delta_{(k)}$ is lower bounded by

$$
\begin{aligned}
\Delta_{(k)} \geq & \Delta_{(k)}^{\mathrm{B}} \\
= & \frac{1-\rho}{2 \mu \rho^{k}(k+1)}\left(\frac{2}{(1-\rho)^{3}}-\sum_{j=0}^{k} \rho^{j}(j+2)(j+1)\right) \\
& -\frac{\rho}{\mu(1-\rho)^{2}(k+1)},
\end{aligned}
$$

which asymptotically approaches $\Delta_{(k)}$ as $\rho \rightarrow 1$.

Proposition 2 (idle case): $\Delta_{(k)}$ is lower bounded by

$$
\Delta_{(k)} \geq \Delta_{(k)}^{\mathrm{I}}=\frac{1}{\lambda}+\frac{k}{2 \lambda}+\frac{1}{\mu},
$$

which asymptotically approaches $\Delta_{(k)}$ as $\rho \rightarrow 0$.

The proofs of Proposition 1 and Proposition 2 are presented in Appendix B and Appendix C, respectively. Now, we verify the lower bounds by numerical results. Consider the simplest case that $k=0$, i.e., no outage event occurs, and then the lower bounds of $\Delta_{(0)}$ can be obtained as

$$
\begin{cases}\Delta_{(0)}^{\mathrm{B}}=\frac{\rho(2-\rho)}{\mu(1-\rho)}, & \text { for busy case, } \\ \Delta_{(0)}^{\mathrm{I}}=\frac{1}{\lambda}+\frac{1}{\mu}, & \text { for idle case. }\end{cases}
$$

Fig. 6(a) compares the lower bound for busy case with the exact AoI $\Delta_{(0)}$ presented in (32). Clearly, the lower bound is always below the exact AoI for arbitrary $\rho$ and $\mu$. Moreover, the lower bound and the exact value of AoI asymptotically converge together as $\rho$ tends to 1 . This observation matches our intuition that the system becomes busier with larger $\rho$, and hence the total service time in (43) becomes closer to $\left(\tilde{y}_{i}+\tilde{t}_{i}\right)$. Consequently, we can use the lower bound for busy case to approximate AoI when $\rho$ is relatively large.

On the other hand, the lower bound for idle case is compared with the exact AoI in Fig. 6(b). It is found that the lower bound and the exact AoI start from the same point when $\rho$ is extremely small. This observation is understood in the following way: the queue will be almost empty if $\rho$ decreases to a relatively small value, and hence the zero waiting time exactly matches the condition for deriving $\Delta_{(k)}^{\mathrm{I}}$. Opposite to Fig. 6(a), the gap between the lower bound and the exact AoI extends as $\rho$ goes larger. Inspired by the different tendency between Fig. 6(a) and Fig. 6(b), we combine the lower bounds for two cases together and obtain a more accurate lower bound, i.e.,

Proposition 3: $\Delta_{(k)}$ is lower bounded by

$$
\Delta_{(k)} \geq \Delta_{(k)}^{\mathrm{LB}}=\max \left(\Delta_{(k)}^{\mathrm{B}}, \Delta_{(k)}^{\mathrm{I}}\right)
$$

Finally, we can substitute (38) into (31) to calculate the lower bound of the average AoI in the communication system where outage events occur.

\section{Simulations}

In order to evaluate how precise the lower bound is, we have conducted a series of simulations to measure the exact AoI. Fig. 7 illustrates the simulation results and the corresponding lower bound. Although there is a gap between the lower bound

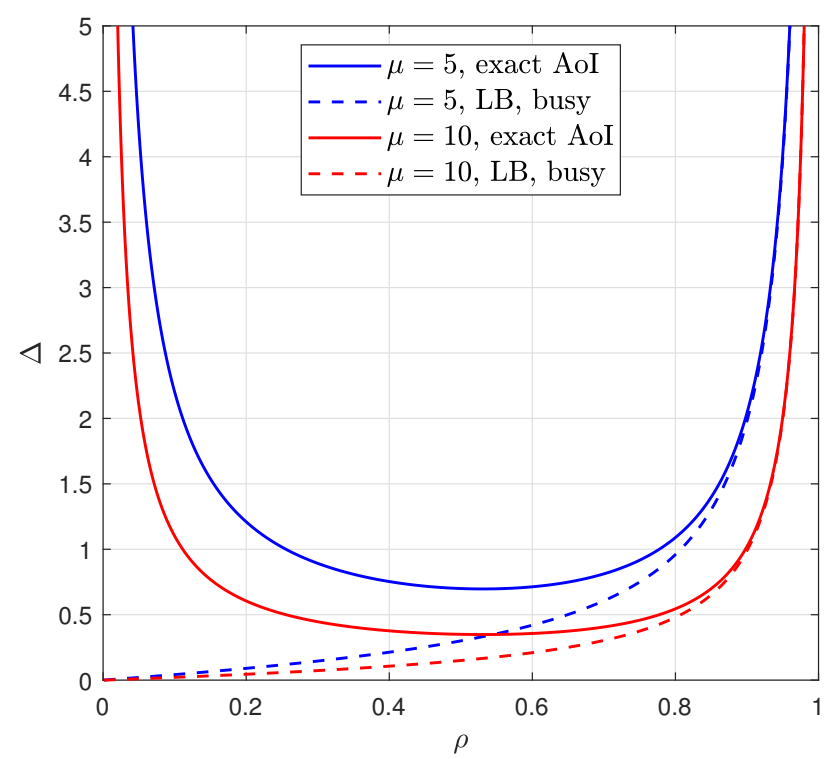

(a) Busy case.

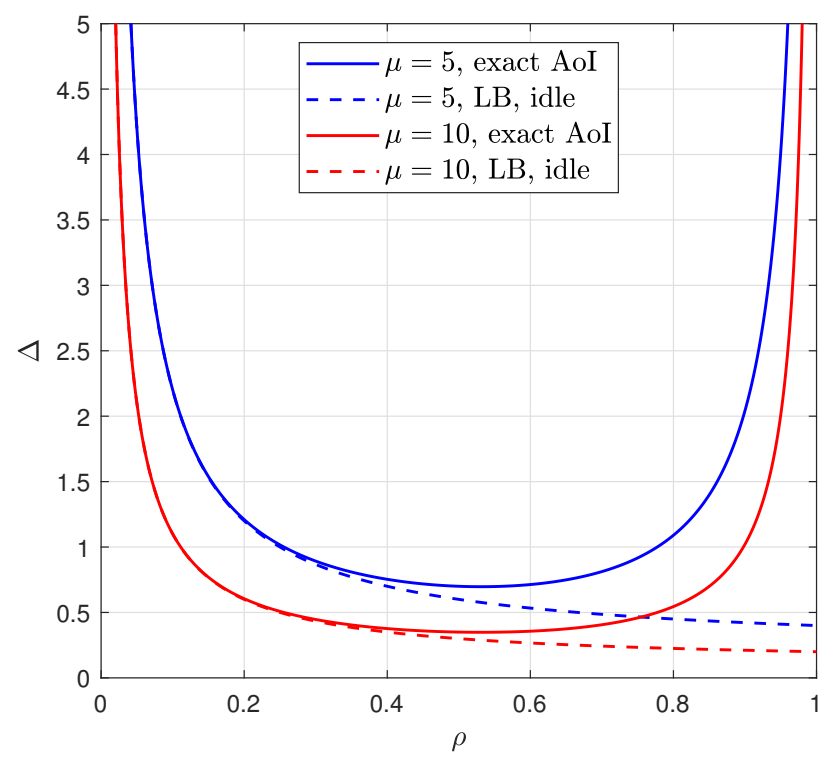

(b) Idle case.

Fig. 6. The exact value versus the lower bound of $\Delta_{(0)}$.

and the simulation result, they have imperceptible differences when $\rho \leq 0.4$ or $\rho \geq 0.9$. From the comparison between Fig. 7(a) and Fig. 7(b), we can make a conclusion that the stronger the service ability, i.e., larger $\mu$, the smaller the gap. In addition, the gap between the lower bound and the simulation result increases with larger outage probability $P_{\text {out }}$. Because the outage event occurs more frequently for larger $P_{\text {out }}$, and more updates are contained in a greater number of continuous outage events. Consequently, the deviation resulting from the idle time and the waiting time also increases for continuous updates. This implies that it may not be sufficiently accurate to estimate the exact AoI by the lower bound for large $P_{\text {out }}$. Nevertheless, notice that the gap for $P_{\text {out }}=0.5$ is still tolerable. Actually, $P_{\text {out }}=0.5$ for practical systems is a quite 


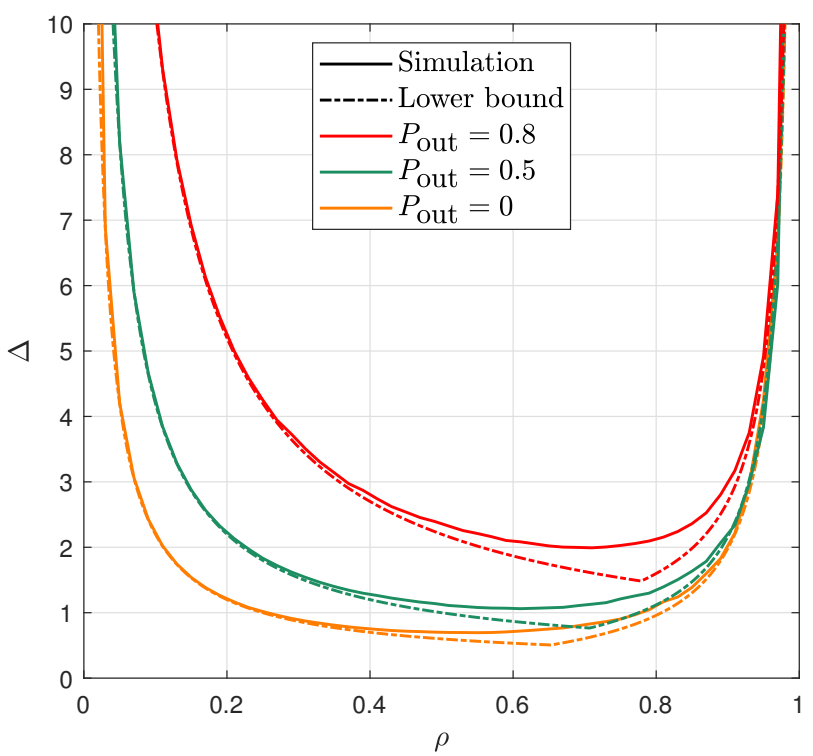

(a) $\mu=5$.

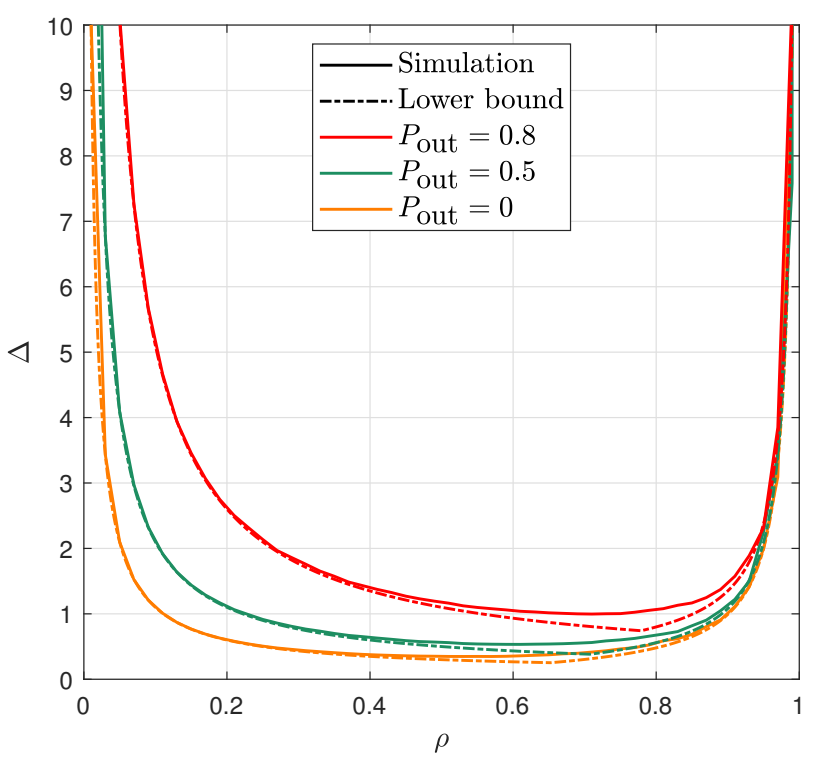

(b) $\mu=10$.

Fig. 7. The comparison between the relatively tight lower bound and simulation results.

large outage probability which means half of the transmissions failed. Therefore, for such an unreliable communication system with large $P_{\text {out }}$, the performance refinement should focus more on how to reduce the outage probability. A simple method is to skip some transmissions and accumulate more energy to the rest of transmissions. By this means, we can reduce the outage probability for the transmitted updates and consume the same energy in total. In the following, we are going to show an interesting instance that the average AoI can be reduced with the same total energy consumption by the intermittent transmission scheme.

We consider the case with the correlation degree $q=0.8$ and the distortion requirements $D_{1}=D_{2}=0.1$. From Fig. 4(b), we can find that the outage probabilities are approx-

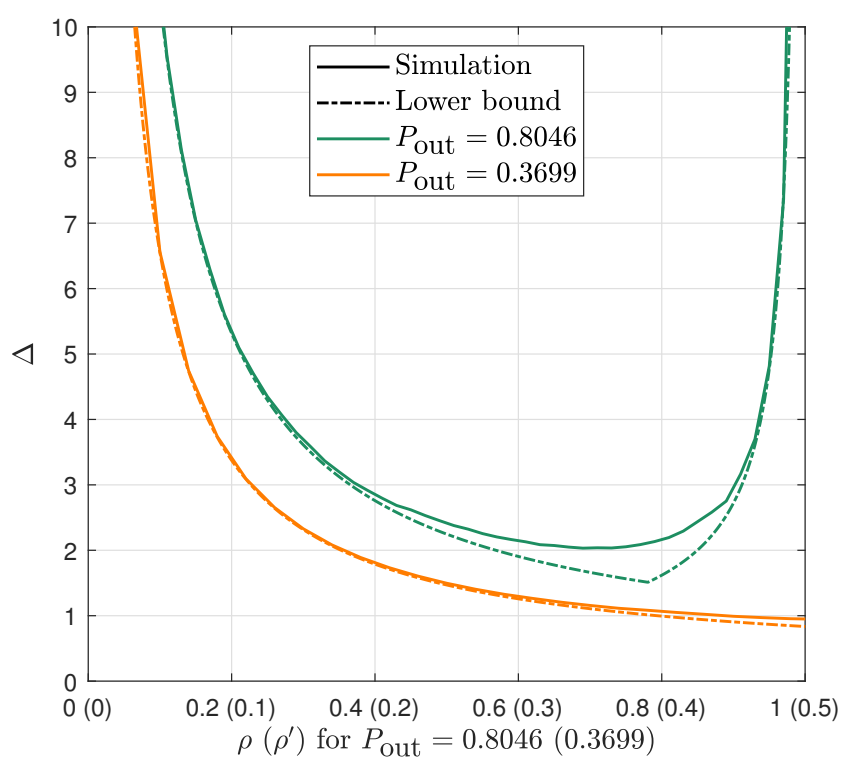

(a) $\mu=5$.

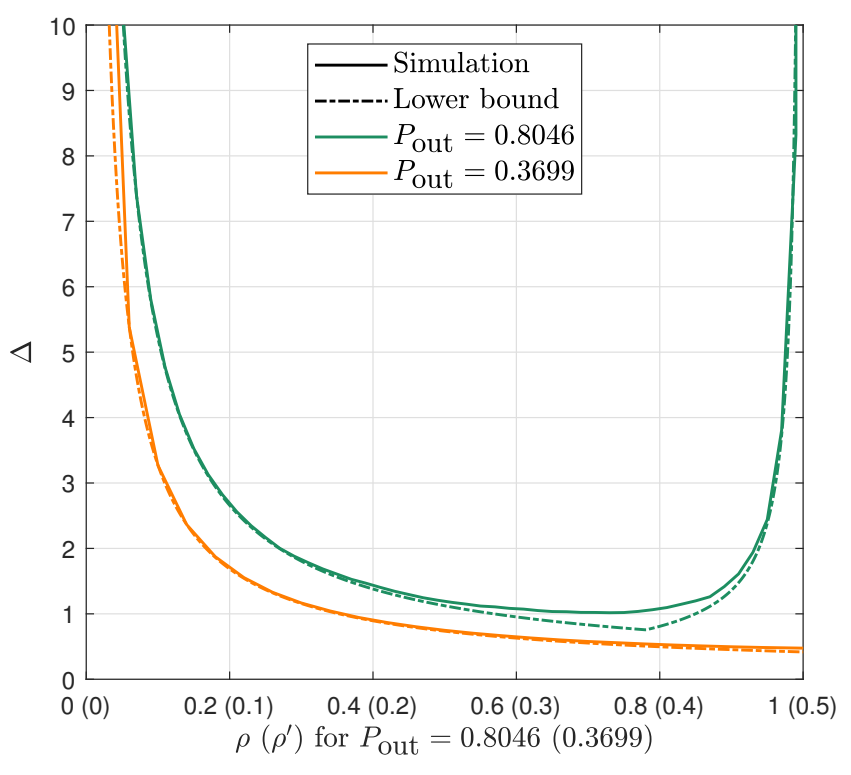

(b) $\mu=10$.

Fig. 8. Refinement of AoI performance.

imately 0.8046 and 0.3699 for $\bar{\gamma}_{i}$ equal to $6.3 \mathrm{~dB}$ and $9.3 \mathrm{~dB}$, respectively. For two continuous updates, the UAV can use two times the power $(+3 \mathrm{~dB})$ for transmitting only one update and ignoring the next update. By this means, the outage probability can be reduced from 0.8046 to 0.3699 , while keeping the total energy consumption unchanged. Then, the equivalent arrival rate for $P_{\text {out }}=0.3699$ reduces to half, and the equivalent server utilization ratio becomes $\rho^{\prime}=\rho / 2$. Fig. 8 depicts the average AoI curves with respect to $\rho$ and $\rho^{\prime}$ for $P_{\text {out }}$ equal to 0.8046 and 0.3699 , respectively. Clearly, the curve with $\rho^{\prime}$ for $P_{\text {out }}=0.3699$ is below that with $\rho$ for $P_{\text {out }}=0.8046$. Thus, both the simulation result and the lower bound verify that the AoI can be refined to a lower level without extra energy consumption by intermittently transmitting the updates for a relatively large outage probability. Moreover, the actual 


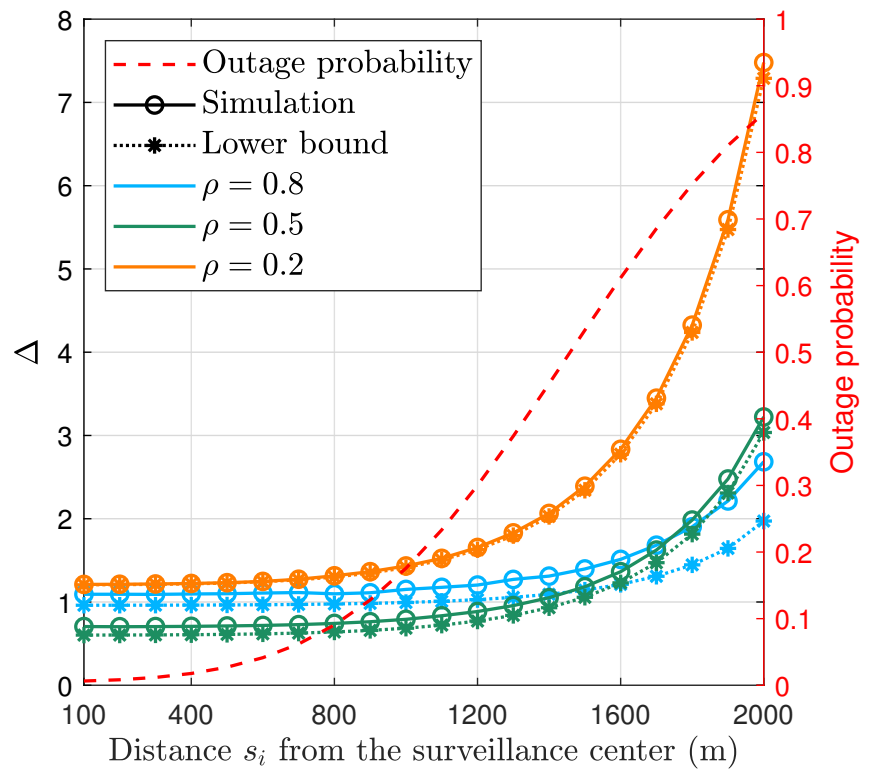

Fig. 9. The average AoI for different UAV locations with $\mu=5$.

performance gain will be more if we perform refinement based on the lower bound, due to the extension of the gap between the lower bound and the exact AoI as $P_{\text {out }}$ increases.

Based on practical parameter settings, we evaluate the effect on the average AoI due to different UAV locations. Assume that the surveillance system is deployed at suburban areas, where the channel conditions are dominated by the impact of environment. Therefore, the average SNR is calculated by (7), and the parameters for calculating path-loss at suburban are $\left(\eta_{\mathrm{LoS}}, \eta_{\mathrm{NLoS}}, a, b\right)=(0.1,21,4.88,0.43)$ [22, Table V]. The altitudes $h_{i}$ of UAVs keep at $500 \mathrm{~m}$ while their distances $s_{i}$ from the surveillance center change from $100 \mathrm{~m}$ to $2000 \mathrm{~m}$. In order to compare the tendencies of the average AoI and the outage probability, we plot the curves in a two-dimensional plane by setting $s_{i}$ at the same value. If the distances from the surveillance center to the UAVs are different, we can also obtain similar conclusions. Although the UAV locations may affect the correlation degree between the information collected by different UAVs, we assume that the correlation degree stays the same at $q=0.8$ for simplicity. We set the distortion requirements $D_{i}=0.1$ and the $\mathrm{UAV}$ transmission power $P_{\mathrm{UAV}, i}=0.5 \mathrm{~W}$. From [42, Eq. (4.2.4)], the thermal noise power is $-174 \mathrm{dBm} / \mathrm{Hz}$ at the room temperature. The carrier frequencies for two UAVs are set at $f_{1}=2 \mathrm{GHz}$ and $f_{2}=1.99$ $\mathrm{GHz}$, respectively. Let the bandwidth be $10 \mathrm{MHz}$, and we can obtain $N_{0}=3.9811 \times 10^{-14} \mathrm{~W}$. As shown in Fig. 9, the average AoI increases obviously when $s_{i}>800 \mathrm{~m}$ where $P_{\text {out }}>0.1$, due to the rapid increase of outage probability. On the contrary, below the $800 \mathrm{~m}$ distance range, the system can keep low level of the average AoI. Moreover, the gap between the lower bound and the exact AoI enlarges only when $\rho=0.8$ and $P_{\text {out }}>0.1$.

\section{CONCLUSION}

We have established an analytical framework for the outage probability and the average AoI of cooperative lossy com- munications in UAV networks. The analytical framework was applied to a UAV cooperative surveillance system for instance. To begin with, we formulated the UAV cooperative surveillance system as a multiterminal source coding problem, and then calculated the outage probability based on the Shannon's lossy source-channel separation theorem. The numerical results verify that the outage probability decreases in cooperative communication systems by joint decoding, and we can observe the diversity gain for relatively large distortion requirements. Subsequently, given a specific value of outage probability, we further derived two lower bounds on the average AoI for the system dominated by the busy and idle states. By combining the lower bounds for the busy and idle cases together, we obtained a more accurate lower bound on the average AoI. Finally, we compared the lower bound with the exact value of AoI by simulations. The simulation results showed that the gap between the lower bound and the exact AoI is imperceptible for relatively busy or idle systems. Moreover, the gap still seems tolerable even for quite large outage probability values in practical systems, i.e., up to $P_{\text {out }}=0.5$, which is well sufficient for practical system performance evaluations. Therefore, we proposed an intermittent transmission scheme to reduce the outage probability while keeping the same total energy consumption. Interestingly, the system can thereby achieve a lower level of both the outage probability and the average AoI without consuming extra energy. We also implemented the analytical framework with practical parameter settings to evaluate the impact on the average AoI due to the change of the UAV locations. Besides UAV cooperative surveillance systems, the framework for the AoI analysis can be also implemented in other multiterminal communication systems accepting outage events. In the future, we are going to apply the analytical framework of the outage probability and the average AoI for correlated fading channels.

\section{APPENDiX A}

DERIVATIONS OF $P_{k}$

The calculations of $P_{k}$ are presented as follows.

$$
\begin{aligned}
P_{1}= & \operatorname{Pr}\left\{A_{1}\right\} \\
= & \operatorname{Pr}\left\{0 \leq R_{1}<\varphi_{1}, R_{2}^{*} \leq R_{2}\right\} \\
= & \operatorname{Pr}\left\{0 \leq \Theta_{1}\left(\gamma_{1}\right)<\varphi_{1}, R_{2}^{*} \leq \Theta_{2}\left(\gamma_{2}\right)\right\} \\
= & \operatorname{Pr}\left\{\Theta_{1}^{-1}(0) \leq \gamma_{1}<\Theta_{1}^{-1}\left(\varphi_{1}\right), \Theta_{2}^{-1}\left(R_{2}^{*}\right) \leq \gamma_{2}\right\} \\
= & \operatorname{Pr}\left\{0 \leq \gamma_{1}<\Theta_{1}^{-1}\left(\varphi_{1}\right), \Theta_{2}^{-1}\left(R_{2}^{*}\right) \leq \gamma_{2}\right\} \\
= & \int_{\Theta_{2}^{-1}\left(R_{2}^{*}\right)}^{\infty} d \gamma_{2} \int_{0}^{\Theta_{1}^{-1}\left(\varphi_{1}\right)} p\left(\gamma_{2}\right) p\left(\gamma_{1}\right) d \gamma_{1} \\
= & \int_{\Theta_{2}^{-1}\left(R_{2}^{*}\right)}^{\infty} d \gamma_{2} \int_{0}^{\Theta_{1}^{-1}\left(\varphi_{1}\right)} p\left(\gamma_{2}\right) \frac{m^{m} \gamma_{1}^{m-1}}{\bar{\gamma}_{1}^{m} \Gamma(m)} \\
& \cdot \exp \left(-\frac{m \gamma_{1}}{\bar{\gamma}_{1}}\right) d \gamma_{1} \\
= & \int_{\Theta_{2}^{-1}\left(R_{2}^{*}\right)}^{\infty} p\left(\gamma_{2}\right)\left[1-\frac{\Gamma\left(m, \frac{m}{\bar{\gamma}_{1}} \Theta_{1}^{-1}\left(\varphi_{1}\right)\right)}{\Gamma(m)} d \gamma_{2} \quad(39)\right. \\
= & \frac{\Gamma\left(m, \frac{m}{\bar{\gamma}_{2}} \Theta_{2}^{-1}\left(R_{2}^{*}\right)\right)}{\Gamma(m)}-\frac{m^{m}}{\bar{\gamma}_{2}^{m}[\Gamma(m)]^{2}} \int_{\Theta_{2}^{-1}\left(R_{2}^{*}\right)}^{\infty} \gamma_{2}^{m-1}
\end{aligned}
$$




$$
\cdot \exp \left(-\frac{m \gamma_{2}}{\bar{\gamma}_{2}}\right) \Gamma\left(m, \frac{m}{\bar{\gamma}_{1}} \Theta_{1}^{-1}\left(\varphi_{1}\right)\right) d \gamma_{2},
$$

where (39) follows according to [43, Eq. (3.381.3)].

$$
\begin{aligned}
& P_{2}=\operatorname{Pr}\left\{A_{2}\right\} \\
& =\operatorname{Pr}\left\{0 \leq R_{1}<\psi-R_{2}, \psi-R_{1}^{*} \leq R_{2}<R_{2}^{*}\right\} \\
& =\operatorname{Pr}\left\{0 \leq \Theta_{1}\left(\gamma_{1}\right)<\psi-\Theta_{2}\left(\gamma_{2}\right)\right. \text {, } \\
& \left.\psi-R_{1}^{*} \leq \Theta_{2}\left(\gamma_{2}\right)<R_{2}^{*}\right\} \\
& =\operatorname{Pr}\left\{\Theta_{1}^{-1}(0) \leq \gamma_{1}<\Theta_{1}^{-1}\left[\psi-\Theta_{2}\left(\gamma_{2}\right)\right]\right. \text {, } \\
& \left.\Theta_{2}^{-1}\left(\psi-R_{1}^{*}\right) \leq \gamma_{2}<\Theta_{2}^{-1}\left(R_{2}^{*}\right)\right\} \\
& =\operatorname{Pr}\left\{0 \leq \gamma_{1}<\Theta_{1}^{-1}\left[\psi-\Theta_{2}\left(\gamma_{2}\right)\right]\right. \text {, } \\
& \left.\Theta_{2}^{-1}\left(\psi-R_{1}^{*}\right) \leq \gamma_{2}<\Theta_{2}^{-1}\left(R_{2}^{*}\right)\right\} \\
& =\int_{\Theta_{2}^{-1}\left(\psi-R_{1}^{*}\right)}^{\Theta_{2}^{-1}\left(R_{2}^{*}\right)} d \gamma_{2} \int_{0}^{\Theta_{1}^{-1}\left[\psi-\Theta_{2}\left(\gamma_{2}\right)\right]} p\left(\gamma_{2}\right) p\left(\gamma_{1}\right) d \gamma_{1} \\
& =\int_{\Theta_{2}^{-1}\left(\psi-R_{1}^{*}\right)}^{\Theta_{2}^{-1}\left(R_{2}^{*}\right)} d \gamma_{2} \int_{0}^{\Theta_{1}^{-1}\left[\psi-\Theta_{2}\left(\gamma_{2}\right)\right]} p\left(\gamma_{2}\right) \frac{m^{m} \gamma_{1}^{m-1}}{\bar{\gamma}_{1}^{m} \Gamma(m)} \\
& \cdot \exp \left(-\frac{m \gamma_{1}}{\bar{\gamma}_{1}}\right) d \gamma_{1} \\
& =\int_{\Theta_{2}^{-1}\left(\psi-R_{1}^{*}\right)}^{\Theta_{2}^{-1}\left(R_{2}^{*}\right)} p\left(\gamma_{2}\right) \\
& \cdot\left[1-\frac{\Gamma\left(m, \frac{m}{\bar{\gamma}_{1}} \Theta_{1}^{-1}\left[\psi-\Theta_{2}\left(\gamma_{2}\right)\right]\right)}{\Gamma(m)}\right] d \gamma_{2} \\
& =\frac{\Gamma\left(m, \frac{m}{\bar{\gamma}_{2}} \Theta_{2}^{-1}\left(\psi-R_{1}^{*}\right)\right)-\Gamma\left(m, \frac{m}{\bar{\gamma}_{2}} \Theta_{2}^{-1}\left(R_{2}^{*}\right)\right)}{\Gamma(m)} \\
& -\frac{m^{m}}{\bar{\gamma}_{2}^{m}[\Gamma(m)]^{2}} \int_{\Theta_{2}^{-1}\left(\psi-R_{1}^{*}\right)}^{\Theta_{2}^{-1}\left(R_{2}^{*}\right)} \gamma_{2}^{m-1} \exp \left(-\frac{m \gamma_{2}}{\bar{\gamma}_{2}}\right) \\
& \cdot \Gamma\left(m, \frac{m}{\bar{\gamma}_{1}} \Theta_{1}^{-1}\left[\psi-\Theta_{2}\left(\gamma_{2}\right)\right]\right) d \gamma_{2} \text {. }
\end{aligned}
$$$$
P_{3}=\operatorname{Pr}\left\{A_{3}\right\}
$$$$
=\operatorname{Pr}\left\{0 \leq R_{1}<R_{1}^{*}, 0 \leq R_{2}<\psi-R_{1}^{*}\right\}
$$$$
=\operatorname{Pr}\left\{0 \leq \Theta_{1}\left(\gamma_{1}\right)<R_{1}^{*}, 0 \leq \Theta_{2}\left(\gamma_{2}\right)<\psi-R_{1}^{*}\right\}
$$$$
=\operatorname{Pr}\left\{\Theta_{1}^{-1}(0) \leq \gamma_{1}<\Theta_{1}^{-1}\left(R_{1}^{*}\right)\right. \text {, }
$$$$
\left.\Theta_{2}^{-1}(0) \leq \gamma_{2}<\Theta_{2}^{-1}\left(\psi-R_{1}^{*}\right)\right\}
$$$$
=\operatorname{Pr}\left\{0 \leq \gamma_{1}<\Theta_{1}^{-1}\left(R_{1}^{*}\right), 0 \leq \gamma_{2}<\Theta_{2}^{-1}\left(\psi-R_{1}^{*}\right)\right\}
$$$$
=\int_{0}^{\Theta_{2}^{-1}\left(\psi-R_{1}^{*}\right)} d \gamma_{2} \int_{0}^{\Theta_{1}^{-1}\left(R_{1}^{*}\right)} p\left(\gamma_{2}\right) p\left(\gamma_{1}\right) d \gamma_{1}
$$$$
=\int_{0}^{\Theta_{2}^{-1}\left(\psi-R_{1}^{*}\right)} d \gamma_{2} \int_{0}^{\Theta_{1}^{-1}\left(R_{1}^{*}\right)} p\left(\gamma_{2}\right) \frac{m^{m} \gamma_{1}^{m-1}}{\bar{\gamma}_{1}^{m} \Gamma(m)}
$$$$
\cdot \exp \left(-\frac{m \gamma_{1}}{\bar{\gamma}_{1}}\right) d \gamma_{1}
$$$$
=\left[1-\frac{\Gamma\left(m, \frac{m}{\bar{\gamma}_{1}} \Theta_{1}^{-1}\left(R_{1}^{*}\right)\right)}{\Gamma(m)}\right] \cdot \int_{0}^{\Theta_{2}^{-1}\left(\psi-R_{1}^{*}\right)} p\left(\gamma_{2}\right) d \gamma_{2}
$$$$
=\left[1-\frac{\Gamma\left(m, \frac{m}{\bar{\gamma}_{1}} \Theta_{1}^{-1}\left(R_{1}^{*}\right)\right)}{\Gamma(m)}\right]
$$

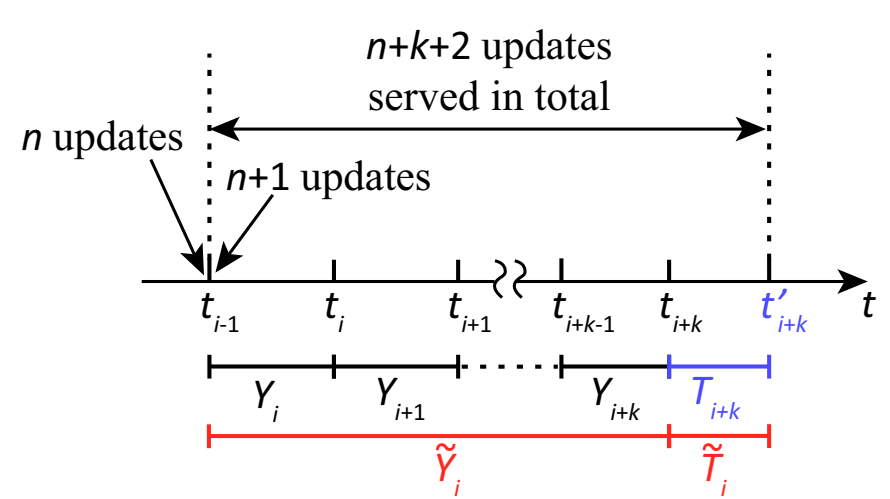

Fig. 10. The number of updates been served from $t_{i-1}$ to $t_{i+k}^{\prime}$.

$$
\cdot\left[1-\frac{\Gamma\left(m, \frac{m}{\bar{\gamma}_{2}} \Theta_{2}^{-1}\left(\psi-R_{1}^{*}\right)\right)}{\Gamma(m)}\right] .
$$

The calculation of $P_{4}$ is symmetric to the calculation of $P_{1}$.

\section{APPENDIX B}

\section{PROOF OF PROPOSITION 1}

We calculate (33) term by term. For $\mathrm{E}\left[\left(\tilde{Y}_{i}+\tilde{T}_{i}\right)^{2}\right]$, we have to find the distribution of $\left(\tilde{Y}_{i}+\tilde{T}_{i}\right)$. As shown in Fig. 10, a new update arrives at $t_{j}$ and its service is finished at $t_{j}^{\prime}$. Assuming that there are $n$ updates in the queue just before $t_{i-1}$, there $(n+k+2)$ updates have been served from $t_{i-1}$ to $t_{i+k}^{\prime}$. The total service time of $(n+k+2)$ updates is

$$
u=u_{1}^{\prime}+\sum_{j=2}^{n+k+2} u_{j}
$$

where $u_{1}^{\prime}$ is the residual service time of an update being served and $u_{j}$ is the service time of the updates waiting in the queue.

If the system is always busy, it means that all updates are continuously served without break from $t_{i-1}$ to $t_{i+k}^{\prime}$, i.e., $u=$ $\tilde{y}_{i}+\tilde{t}_{i}$ in this case. However, the system may be idle during $\left[t_{i-1}, t_{i+k}\right]$, and therefore, $u \leq \tilde{y}_{i}+\tilde{t}_{i}$ in general. Obviously, $U$ is the lower bound of $\left(\tilde{Y}_{i}+\tilde{T}_{i}\right)$. According to [38, Eq. (16-96)], given $n, u$ follows the gamma distribution as

$$
p_{U \mid n}(u)=\frac{\mu^{n+k+2} u^{n+k+1}}{(n+k+1) !} e^{-\mu u} .
$$

The probability for $n$ updates in the queue is $p_{n}=(1-\rho) \rho^{n}$ [38, Eq. (16-88)]. Hence, the distribution of $u$ is

$$
\begin{aligned}
p_{U}(u)= & \sum_{n=0}^{\infty} p_{n} \cdot p_{U \mid n}(u) \\
= & \sum_{n=0}^{\infty}(1-\rho) \rho^{n} \frac{\mu^{n+k+2} u^{n+k+1}}{(n+k+1) !} e^{-\mu u} \\
= & \sum_{n=-k-1}^{\infty}(1-\rho) \rho^{n} \frac{\mu^{n+k+2} u^{n+k+1}}{(n+k+1) !} e^{-\mu u} \\
& -\sum_{n=-k-1}^{-1}(1-\rho) \rho^{n} \frac{\mu^{n+k+2} u^{n+k+1}}{(n+k+1) !} e^{-\mu u} .
\end{aligned}
$$


Consider the first term of (45) as

$$
\begin{aligned}
& \sum_{n=-k-1}^{\infty}(1-\rho) \rho^{n} \frac{\mu^{n+k+2} u^{n+k+1}}{(n+k+1) !} e^{-\mu u} \\
& =\sum_{n^{\prime}=0}^{\infty} \frac{(1-\rho)}{\rho^{k+1}} \rho^{n^{\prime}} \frac{\mu^{n^{\prime}+1} u^{n^{\prime}}}{n^{\prime} !} e^{-\mu u} \\
& =\frac{(1-\rho) \mu}{\rho^{k+1}} e^{-\mu u} \sum_{n^{\prime}=0}^{\infty} \frac{\rho^{n^{\prime}} \mu^{n^{\prime}} u^{n^{\prime}}}{n^{\prime} !} \\
& =\frac{(1-\rho) \mu}{\rho^{k+1}} e^{-\mu(1-\rho) u} .
\end{aligned}
$$

For the second term of (45), we have

$$
\begin{aligned}
& \sum_{n=-k-1}^{-1}(1-\rho) \rho^{n} \frac{\mu^{n+k+2} u^{n+k+1}}{(n+k+1) !} e^{-\mu u} \\
& =\sum_{n^{\prime}=0}^{k} \frac{(1-\rho)}{\rho^{k+1}} \rho^{n^{\prime}} \frac{\mu^{n^{\prime}+1} u^{n^{\prime}}}{\left(n^{\prime}\right) !} e^{-\mu u} \\
& =\frac{(1-\rho) \mu}{\rho^{k+1}} e^{-\mu u} \sum_{n^{\prime}=0}^{k} \frac{(\rho \mu u)^{n^{\prime}}}{n^{\prime} !} .
\end{aligned}
$$

Consequently, the PDF of $U$ is given by

$$
p_{U}(u)=\frac{(1-\rho) \mu}{\rho^{k+1}}\left(e^{-\mu(1-\rho) u}-e^{-\mu u} \sum_{n^{\prime}=0}^{k} \frac{(\rho \mu u)^{n^{\prime}}}{n^{\prime} !}\right) .
$$

Now, we can calculate $\mathrm{E}\left[U^{2}\right]$ as follows

$$
\begin{aligned}
\mathrm{E}\left[U^{2}\right]= & \int_{0}^{\infty} u^{2} p_{U}(u) d u \\
= & \int_{0}^{\infty} u^{2} \frac{(1-\rho) \mu}{\rho^{k+1}} \\
& \cdot\left(e^{-\mu(1-\rho) u}-e^{-\mu u} \sum_{n^{\prime}=0}^{k} \frac{(\rho \mu u)^{n^{\prime}}}{n^{\prime} !}\right) d u \\
= & \frac{(1-\rho) \mu}{\rho^{k+1}} \\
& \cdot \int_{0}^{\infty}\left(u^{2} e^{-\mu(1-\rho) u}-\sum_{n^{\prime}=0}^{k} \frac{(\rho \mu)^{n^{\prime}}}{n^{\prime} !} u^{n^{\prime}+2} e^{-\mu u}\right) d u .
\end{aligned}
$$

According to [43, Eq. (2.321.2)], we have

$$
\begin{aligned}
& \int_{0}^{\infty} u^{2} e^{-\mu(1-\rho) u} d u \\
& \quad=\left.e^{-\mu(1-\rho) u}\left(\sum_{j=0}^{2} \frac{-j !\left(\begin{array}{c}
2 \\
j
\end{array}\right)}{[\mu(1-\rho)]^{j+1}} u^{2-j}\right)\right|_{u=0} ^{\infty} \\
& \quad=\frac{2}{\mu^{3}(1-\rho)^{3}} .
\end{aligned}
$$

Similarly, we have

$$
\begin{aligned}
& \int_{0}^{\infty} \sum_{n^{\prime}=0}^{k} \frac{(\rho \mu)^{n^{\prime}}}{n^{\prime} !} u^{n^{\prime}+2} e^{-\mu u} d u \\
& =\sum_{n^{\prime}=0}^{k} \frac{(\rho \mu)^{n^{\prime}}}{n^{\prime} !} \int_{0}^{\infty} u^{n^{\prime}+2} e^{-\mu u} d u \\
& =\left.\sum_{n^{\prime}=0}^{k} \frac{(\rho \mu)^{n^{\prime}}}{n^{\prime} !} e^{-\mu u}\left(\sum_{j=0}^{n^{\prime}+2} \frac{-j !\left(\begin{array}{c}
n^{\prime}+2 \\
j
\end{array}\right)}{\mu^{j+1}} u^{n^{\prime}+2-j}\right)\right|_{u=0} ^{\infty} \\
& =\sum_{n^{\prime}=0}^{k} \frac{(\rho \mu)^{n^{\prime}}}{n^{\prime} !} \cdot \frac{\left(n^{\prime}+2\right) !}{\mu^{n^{\prime}+3}} \\
& =\sum_{n^{\prime}=0}^{k} \frac{\rho^{n^{\prime}}\left(n^{\prime}+2\right)\left(n^{\prime}+1\right)}{\mu^{3}}
\end{aligned}
$$

Hence, we can finally obtain the closed-form expression of $\mathrm{E}\left[U^{2}\right]$ as

$$
\begin{aligned}
\mathrm{E}\left[U^{2}\right]= & \frac{(1-\rho) \mu}{\rho^{k+1}} \\
& \cdot\left(\frac{2}{\mu^{3}(1-\rho)^{3}}-\sum_{n^{\prime}=0}^{k} \frac{\rho^{n^{\prime}}\left(n^{\prime}+2\right)\left(n^{\prime}+1\right)}{\mu^{3}}\right) \\
= & \frac{1-\rho}{\mu^{2} \rho^{k+1}}\left(\frac{2}{(1-\rho)^{3}}-\sum_{j=0}^{k} \rho^{j}(j+2)(j+1)\right) .
\end{aligned}
$$

For $\mathrm{E}\left[\tilde{T}_{i}^{2}\right]$, the PDF of the system time $T$ is given by $[38$, Eq. (16-97)]

$$
p_{T}(t)=\mu(1-\rho) e^{-\mu(1-\rho) t} .
$$

Then, we have

$$
\begin{aligned}
\mathrm{E}\left[\tilde{T}_{i}^{2}\right] & =\mathrm{E}\left[T_{i+k}^{2}\right] \\
& =\mathrm{E}\left[T^{2}\right] \\
& =\int_{0}^{\infty} t^{2} p_{T}(t) d t \\
& =\int_{0}^{\infty} t^{2} \mu(1-\rho) e^{-\mu(1-\rho) t} d t \\
& =\mu(1-\rho) \int_{0}^{\infty} t^{2} e^{-\mu(1-\rho) t} d t \\
& =\left.\mu(1-\rho) e^{-\mu(1-\rho) t} \cdot\left(\sum_{j=0}^{2} \frac{-j !\left(\begin{array}{c}
2 \\
j
\end{array}\right)}{[\mu(1-\rho)]^{j+1}} t^{2-j}\right)\right|_{t=0} ^{\infty} \\
& =\mu(1-\rho) \cdot \frac{2}{\mu^{3}(1-\rho)^{3}} \\
& =\frac{2}{\mu^{2}(1-\rho)^{2}},
\end{aligned}
$$

where (54) follows according to [43, Eq. (2.321.2)].

Regarding $\mathrm{E}\left[\tilde{Y}_{i}\right]$, since the arrival of update follows a Poisson process with mean arrival rate $\lambda$, the arrival time $Y_{i}$ 
follows independent and identically distributed (i.i.d.) exponential distribution with parameter $\lambda$. Therefore, $\mathrm{E}\left[Y_{i}\right]=\frac{1}{\lambda}$, and

$$
\mathrm{E}\left[\tilde{Y}_{i}\right]=\mathrm{E}\left[\sum_{j=i}^{i+k} Y_{j}\right]=\sum_{j=i}^{i+k} \mathrm{E}\left[Y_{j}\right]=\frac{k+1}{\lambda}
$$

Now, we substitute the results of each term into (33) as

$$
\begin{aligned}
\Delta_{(k)}= & \frac{\mathrm{E}\left[\left(\tilde{Y}_{i}+\tilde{T}_{i}\right)^{2}\right]-\mathrm{E}\left[\tilde{T}_{i}^{2}\right]}{2 \mathrm{E}\left[\tilde{Y}_{i}\right]} \\
\geq & \frac{\mathrm{E}\left[U^{2}\right]-\mathrm{E}\left[\tilde{T}_{i}^{2}\right]}{2 \mathrm{E}\left[\tilde{Y}_{i}\right]} \\
= & \frac{1-\rho}{\mu^{2} \rho^{k+1}}\left(\frac{2}{(1-\rho)^{3}}-\sum_{j=0}^{k} \rho^{j}(j+2)(j+1)\right) \\
& \cdot \frac{\lambda}{2(k+1)}-\frac{2}{\mu^{2}(1-\rho)^{2}} \cdot \frac{\lambda}{2(k+1)} \\
= & \frac{1-\rho}{2 \mu \rho^{k}(k+1)}\left(\frac{2}{(1-\rho)^{3}}-\sum_{j=0}^{k} \rho^{j}(j+2)(j+1)\right. \\
& -\frac{\rho}{\mu(1-\rho)^{2}(k+1)} .
\end{aligned}
$$

Consequently, (57) is the lower bound of $\Delta_{(k)}$ for busy case, and this finishes the proof of Proposition 1.

\section{APPENDIX C}

\section{PROOF OF PROPOSITION 2}

Proof: Converse to the busy case, we consider the opposite extreme case, i.e., the system is completely idle and hence the queue is empty. In this case, the waiting time of each update is 0 . Consider

$$
\begin{aligned}
\Delta_{(k)} & =\frac{\mathrm{E}\left[\left(\tilde{Y}_{i}+\tilde{T}_{i}\right)^{2}\right]-\mathrm{E}\left[\tilde{T}_{i}^{2}\right]}{2 \mathrm{E}\left[\tilde{Y}_{i}\right]} \\
& =\frac{\mathrm{E}\left[\tilde{Y}_{i}^{2}\right]+2 \mathrm{E}\left[\tilde{Y}_{i} \tilde{T}_{i}\right]}{2 \mathrm{E}\left[\tilde{Y}_{i}\right]} \\
& =\frac{\mathrm{E}\left[\tilde{Y}_{i}^{2}\right]+2 \mathrm{E}\left[\sum_{j=i}^{i+k} Y_{j} \cdot T_{i+k}\right]}{2 \mathrm{E}\left[\tilde{Y}_{i}\right]} \\
& =\frac{\mathrm{E}\left[\tilde{Y}_{i}^{2}\right]+2 \sum_{j=i}^{i+k} \mathrm{E}\left[Y_{j} T_{i+k}\right]}{2 \mathrm{E}\left[\tilde{Y}_{i}\right]} \\
& =\frac{\mathrm{E}\left[\tilde{Y}_{i}^{2}\right]+2 \sum_{j=i}^{i+k} \mathrm{E}\left[Y_{j}\left(W_{i+k}+S_{i+k}\right)\right]}{2 \mathrm{E}\left[\tilde{Y}_{i}\right]} \\
& \geq \frac{\mathrm{E}\left[\tilde{Y}_{i}^{2}\right]+2 \sum_{j=i}^{i+k} \mathrm{E}\left[Y_{j} S_{i+k}\right]}{2 \mathrm{E}\left[\tilde{Y}_{i}\right]} \\
& =\frac{\mathrm{E}\left[\tilde{Y}_{i}^{2}\right]}{2 \mathrm{E}\left[\tilde{Y}_{i}\right]}+\frac{2 \sum_{j=i}^{i+k} \mathrm{E}\left[Y_{j}\right] \mathrm{E}\left[S_{i+k}\right]}{2 \sum_{j=i}^{i+k} \mathrm{E}\left[Y_{j}\right]} \\
& =\frac{\mathrm{E}\left[\tilde{Y}_{i}^{2}\right]}{2 \mathrm{E}\left[\tilde{Y}_{i}\right]}+\mathrm{E}\left[S_{i+k}\right],
\end{aligned}
$$

where the equality of (58) holds when the system is completely idle. Therefore, the lower bound for idle case can be further derived from (59). We can calculate the first term of (59) as

$$
\begin{aligned}
\frac{\mathrm{E}\left[\tilde{Y}_{i}^{2}\right]}{2 \mathrm{E}\left[\tilde{Y}_{i}\right]}= & \frac{\mathrm{E}\left[\left(\sum_{j=i}^{i+k} Y_{j}\right)^{2}\right]}{2 \mathrm{E}\left[\sum_{j=i}^{i+k} Y_{j}\right]} \\
& =\frac{\mathrm{E}\left[\sum_{j=i}^{i+k} Y_{j}^{2}+2 \sum_{j=i}^{i+k-1} \sum_{j^{\prime}=j+1}^{i+k} Y_{j} Y_{j^{\prime}}\right]}{2 \sum_{j=i}^{i+k} \mathrm{E}\left[Y_{j}\right]} \\
& =\frac{(k+1) \mathrm{E}\left[Y^{2}\right]+2 \sum_{j=i}^{i+k-1} \sum_{j^{\prime}=j+1}^{i+k} \mathrm{E}\left[Y_{j}\right] \mathrm{E}\left[Y_{j^{\prime}}\right]}{2(k+1) \mathrm{E}\left[Y^{\prime}\right]} \\
& =\frac{(k+1) \mathrm{E}\left[Y^{2}\right]+k(k+1)(\mathrm{E}[Y])^{2}}{2(k+1) \mathrm{E}[Y]} \\
& =\frac{\mathrm{E}\left[Y^{2}\right]+k(\mathrm{E}[Y])^{2}}{2 \mathrm{E}[Y]} .
\end{aligned}
$$

It is easy to obtain $\mathrm{E}\left[Y^{2}\right]=\frac{2}{\lambda^{2}}$ for exponential distribution. Hence, we have

$$
\frac{\mathrm{E}\left[\tilde{Y}_{i}^{2}\right]}{2 \mathrm{E}\left[\tilde{Y}_{i}\right]}=\frac{1}{\lambda}+\frac{k}{2 \lambda} .
$$

By substituting (61) and $\mathrm{E}[S]=\frac{1}{\mu}$ into (59), we can obtain the lower bound of $\Delta_{(k)}$ for idle case as

$$
\Delta_{(k)} \geq \frac{1}{\lambda}+\frac{k}{2 \lambda}+\frac{1}{\mu} .
$$

This finishes the proof of Proposition 2.

\section{REFERENCES}

[1] H. Wang, H. Zhao, J. Zhang, D. Ma, J. Li, and J. Wei, "Survey on unmanned aerial vehicle networks: A cyber physical system perspective," IEEE Communications Surveys \& Tutorials, vol. 22, no. 2, pp. 10271070, 2020.

[2] L. Li, H. Ren, Q. Cheng, K. Xue, W. Chen, M. Debbah, and Z. Han, "Millimeter-wave networking in the sky: A machine learning and mean field game approach for joint beamforming and beam-steering," IEEE Transactions on Wireless Communications, vol. 19, no. 10, pp. 6393 6408, Oct. 2020.

[3] H. Zhang, J. Zhang, and K. Long, "Energy efficiency optimization for NOMA UAV network with imperfect CSI," IEEE Journal on Selected Areas in Communications, vol. 38, no. 12, pp. 2798-2809, Dec. 2020.

[4] L. Li, Y. Sun, Q. Cheng, D. Wang, W. Lin, and W. Chen, "Optimal trajectory and downlink power control for multi-type UAV aerial base stations," Chinese Journal of Aeronautics, Early Access, DOI: 10.1016/j.cja.2020.12.019, 2021.

[5] L. Li, Y. Xu, Z. Zhang, J. Yin, W. Chen, and Z. Han, "A predictionbased charging policy and interference mitigation approach in the wireless powered internet of things," IEEE Journal on Selected Areas in Communications, vol. 37, no. 2, pp. 439-451, Feb. 2019.

[6] Z. Xiong, Y. Zhang, W. Y. B. Lim, J. Kang, D. Niyato, C. Leung, and C. Miao, "UAV-assisted wireless energy and data transfer with deep reinforcement learning," IEEE Transactions on Cognitive Communications and Networking, vol. 7, no. 1, pp. 85-99, 2021.

[7] W. Y. B. Lim, J. Huang, Z. Xiong, J. Kang, D. Niyato, X.-S. Hua, C. Leung, and C. Miao, "Towards federated learning in UAV-enabled internet of vehicles: A multi-dimensional contract-matching approach," IEEE Transactions on Intelligent Transportation Systems, Early Access, DOI: 10.1109/TITS.2021.3056341, 2021

[8] A. Kosta, N. Pappas, and V. Angelakis, Age of Information: A New Concept, Metric, and Tool. Hanover, MA: now Publishers Inc., 2017.

[9] Q. He, D. Yuan, and A. Ephremides, "Optimizing freshness of information: On minimum age link scheduling in wireless systems," in 14th International Symposium on Modeling and Optimization in Mobile, Ad Hoc, and Wireless Networks (WiOpt), Tempe, AZ, May 2016. 
[10] —, "On optimal link scheduling with min-max peak age of information in wireless systems," in IEEE International Conference on Communications (ICC), Kuala Lumpur, Malaysia, May 2016.

[11] R. D. Yates, "Lazy is timely: Status updates by an energy harvesting source," in IEEE International Symposium on Information Theory (ISIT), Hong Kong, China, Jun. 2015, pp. 3008-3012.

[12] B. T. Bacinoglu, E. T. Ceran, and E. Uysal-Biyikoglu, "Age of information under energy replenishment constraints," in Information Theory and Applications Workshop (ITA), San Diego, CA, Feb. 2015, pp. 25-31.

[13] S. Zhang, H. Zhang, L. Song, Z. Han, and H. V. Poor, "Sensing and communication tradeoff design for AoI minimization in a cellular internet of UAVs," in IEEE International Conference on Communications, Dublin, Ireland, Jun. 2020.

[14] J. N. Laneman, D. N. Tse, and G. W. Wornell, "Cooperative diversity in wireless networks: Efficient protocols and outage behavior," IEEE Transactions on Information Theory, vol. 50, no. 12, pp. 3062-3080, Dec. 2004.

[15] J. He, V. Tervo, X. Zhou, X. He, S. Qian, M. Cheng, M. Juntti, and T. Matsumoto, "A tutorial on lossy forwarding cooperative relaying," IEEE Communications Surveys \& Tutorials, vol. 21, no. 1, pp. 66-87, First quarter 2018.

[16] X. Zhou, M. Cheng, X. He, and T. Matsumoto, "Exact and approximated outage probability analyses for decode-and-forward relaying system allowing intra-link errors," IEEE Transactions on Wireless Communications, vol. 13, no. 12, pp. 7062-7071, Dec. 2014

[17] S. Qian, X. Zhou, X. He, J. He, M. Juntti, and T. Matsumoto, "Performance analysis for lossy-forward relaying over Nakagami- $m$ fading channels," IEEE Transactions on Vehicular Technology, vol. 66, no. 11, pp. 10035-10043, Nov. 2017.

[18] W. Lin, S. Qian, and T. Matsumoto, "Lossy-forward relaying for lossy communications: Rate-distortion and outage probability analyses," IEEE Transactions on Wireless Communications, vol. 18, no. 8, pp. 3974 3986, Aug. 2019.

[19] S. Qian, J. He, M. Juntti, and T. Matsumoto, "Fading correlations for wireless cooperative communications: Diversity and coding gains," IEEE Access, vol. 5, pp. 8001-8016, Apr. 2017.

[20] P.-S. Lu, X. Zhou, and T. Matsumoto, "Outage probabilities of orthogonal multiple-access relaying techniques with imperfect source-relay links," IEEE Transactions on Wireless Communications, vol. 14, no. 4, pp. 2269-2280, Apr. 2015.

[21] W. Lin, Q. Xue, J. He, M. Juntti, and T. Matsumoto, "Rate-distortion and outage probability analyses for single helper assisted lossy communications," IEEE Transactions on Vehicular Technology, vol. 68, no. 11, pp. 10882-10 894, Nov. 2019.

[22] A. A. Khuwaja, Y. Chen, N. Zhao, M. Alouini, and P. Dobbins, "A survey of channel modeling for UAV communications," IEEE Communications Surveys \& Tutorials, vol. 20, no. 4, pp. 2804-2821, Fourth quarter 2018.

[23] S. Kaul, M. Gruteser, V. Rai, and J. Kenney, "Minimizing age of information in vehicular networks," in 8th Annual IEEE Communications Society Conference on Sensor, Mesh and Ad Hoc Communications and Networks, Salt Lake City, UT, Jun. 2011, pp. 350-358.

[24] S. Kaul, R. Yates, and M. Gruteser, "Real-time status: How often should one update?" in IEEE Conference on Computer Communications (INFOCOM), Orlando, FL, Mar. 2012, pp. 2731-2735.

[25] S. K. Kaul, R. D. Yates, and M. Gruteser, "Status updates through queues," in 46th Annual Conference on Information Sciences and Systems (CISS), Princeton, NJ, Mar. 2012.

[26] R. Han, J. Wang, L. Bai, J. Liu, and J. Choi, "Age of information and performance analysis for UAV-aided IoT systems," IEEE Internet of Things Journal, Early Access, DOI: 10.1109/JIOT.2021.3051361, 2021.

[27] G. Ahani, D. Yuan, and Y. Zhao, "Age-optimal UAV scheduling for data collection with battery recharging," IEEE Communications Letters, pp. $1-1,2020$.

[28] M. A. Abd-Elmagid and H. S. Dhillon, "Average peak age-ofinformation minimization in UAV-assisted IoT networks," IEEE Transactions on Vehicular Technology, vol. 68, no. 2, pp. 2003-2008, 2019.

[29] K. Chen and L. Huang, "Age-of-information in the presence of error," in 2016 IEEE International Symposium on Information Theory (ISIT), Barcelona, Spain, Jul. 2016, pp. 2579-2583.

[30] S. Zhang, H. Zhang, Z. Han, H. V. Poor, and L. Song, "Age of information in a cellular internet of UAVs: Sensing and communication tradeoff design," IEEE Transactions on Wireless Communications, vol. 19, no. 10, pp. 6578-6592, Oct. 2020

[31] Y. Gu, H. Chen, Y. Zhou, Y. Li, and B. Vucetic, "Timely status update in internet of things monitoring systems: An age-energy tradeoff," IEEE Internet of Things Journal, vol. 6, no. 3, pp. 5324-5335, 2019.
[32] Y. Gu, H. Chen, C. Zhai, Y. Li, and B. Vucetic, "Minimizing age of information in cognitive radio-based IoT systems: Underlay or overlay?" IEEE Internet of Things Journal, vol. 6, no. 6, pp. 10273-10288, 2019.

[33] C. E. Shannon, "A mathematical theory of communication," Bell System Technical Journal, vol. 27, no. 3, pp. 379-423, Jul. 1948.

[34] _ " "Coding theorems for a discrete source with a fidelity criterion," IRE Nat. Conv. Rec, vol. 4, no. 142-163, p. 1, Mar. 1959.

[35] J. Garcia-Frias and Y. Zhao, "Near-Shannon/Slepian-Wolf performance for unknown correlated sources over AWGN channels," IEEE Transactions on Communications, vol. 53, no. 4, pp. 555-559, Apr. 2005.

[36] A. A. Khuwaja, Y. Chen, and G. Zheng, "Effect of user mobility and channel fading on the outage performance of UAV communications," IEEE Wireless Communications Letters, vol. 9, no. 3, pp. 367-370, Mar. 2020.

[37] A. Al-Hourani, S. Kandeepan, and S. Lardner, "Optimal LAP altitude for maximum coverage," IEEE Wireless Communications Letters, vol. 3, no. 6, pp. 569-572, Dec. 2014.

[38] A. Papoulis and S. U. Pillai, Probability, random variables, and stochastic processes, 4th ed. New York: McGraw-Hill, 2002.

[39] Y. Oohama, "Gaussian multiterminal source coding," IEEE Transactions on Information Theory, vol. 43, no. 6, pp. 1912-1923, Nov. 1997.

[40] A. El Gamal and Y.-H. Kim, Network information theory. Cambridge University Press, 2011.

[41] T. M. Cover and J. A. Thomas, Elements of information theory. John Wiley \& Sons, 2012

[42] Q. Gu, RF system design of transceivers for wireless communications. New York, NY: Springer Science \& Business Media, 2005.

[43] I. S. Gradshteyn and I. M. Ryzhik, Table of integrals, series, and products, 7th ed., A. Jeffrey and D. Zwillinger, Eds. Burlington, MA: Academic Press, 2007.

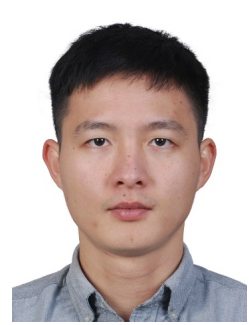

Wensheng Lin (S'17-M'20) received the B.Eng. degree in communication engineering, and the M.Eng. degree in electronic and communication engineering from Northwestern Polytechnical University, Xi'an, China, in 2013 and 2016, respectively, and the $\mathrm{Ph} . \mathrm{D}$. degree in information science from Japan Advanced Institute of Science and Technology (JAIST), Ishikawa, Japan, in 2019. He is currently an Associate Professor with the School of Electronics and Information, Northwestern Polytechnical University, Xi' an, China. His research interests include network information theory, distributed source coding, and Age of Information.

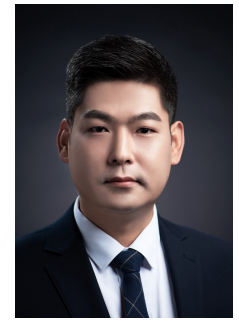

Lixin Li (M'12) received the B.S., the M.S. and the Ph.D. degrees from Northwestern Polytechnical University (NPU), Xi'an, China, in 2001, 2004, and 2008, respectively. He was a Postdoctoral Fellow with NPU from 2009 to 2011. In 2017, He was a Visiting Scholar with the University of Houston, Texas. He is currently an Associate Professor with the School of Electronics and Information, NPU. He has authored or coauthored more than 150 peerreviewed papers in many prestigious journals and conferences, and he holds 20 patents. His current research interests include wireless communications, game theory, and machine learning. He received the 2016 NPU Outstanding Young Teacher Award, which is the highest research and education honors for young faculties in NPU. He was an exemplary reviewer for IEEE Transactions on Communications in 2020 . 


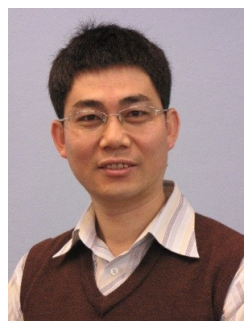

Jinhong Yuan (M'02-SM'11-F'16) received the B.E. and Ph.D. degrees in electronics engineering from the Beijing Institute of Technology, Beijing, China, in 1991 and 1997, respectively. From 1997 to 1999 , he was a Research Fellow with the School of Electrical Engineering, University of Sydney, Sydney, Australia. In 2000, he joined the School of Electrical Engineering and Telecommunications, University of New South Wales, Sydney, Australia, where he is currently a Professor and Head of Telecommunication Group with the School. He has published two books, five book chapters, over 300 papers in telecommunications journals and conference proceedings, and 50 industrial reports. He is a co-inventor of one patent on MIMO systems and two patents on lowdensity-parity-check codes. He has co-authored four Best Paper Awards and one Best Poster Award, including the Best Paper Award from the IEEE International Conference on Communications, Kansas City, USA, in 2018 , the Best Paper Award from IEEE Wireless Communications and Networking Conference, Cancun, Mexico, in 2011, and the Best Paper Award from the IEEE International Symposium on Wireless Communications Systems, Trondheim, Norway, in 2007. He is an IEEE Fellow and currently serving as an Associate Editor for the IEEE Transactions on Wireless Communications and IEEE Transactions on Communications. He served as the IEEE NSW Chapter Chair of Joint Communications/Signal Processions/Ocean Engineering Chapter during 2011-2014 and served as an Associate Editor for the IEEE Transactions on Communications during 2012-2017. His current research interests include error control coding and information theory, communication theory, and wireless communications.

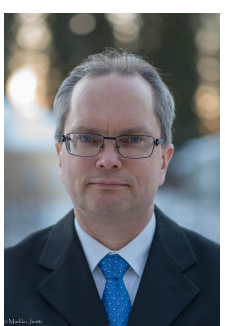

Markku Juntti (S'93-M'98-SM'04-F'20) received his M.Sc. (EE) and Dr.Sc. (EE) degrees from University of Oulu, Oulu, Finland in 1993 and 1997, respectively.

Dr. Juntti was with University of Oulu in 1992-98. In academic year 1994-95, he was a Visiting Scholar at Rice University, Houston, Texas. In 1999-2000, he was a Senior Specialist with Nokia Networks in Oulu, Finland. Dr. Juntti has been a professor of communications engineering since 2000 at University of Oulu, Centre for Wireless Communications (CWC), where he leads the Communications Signal Processing (CSP) Research Group. He also serves as Head of CWC - Radio Technologies (RT) Research Unit. His research interests include signal processing for wireless networks as well as communication and information theory. He is an author or co-author in almost 500 papers published in international journals and conference records as well as in books Wideband CDMA for UMTS in 2000-2010, Handbook of Signal Processing Systems in 2013 and 2018 and $5 G$ Wireless Technologies in 2017. Dr. Juntti is also an Adjunct Professor at Department of Electrical and Computer Engineering, Rice University, Houston, Texas, USA.

Dr. Juntti was an Editor of IEEE TRANSACTIONS ON CommunicaTIONS and an Associate Editor for IEEE TRANSACTIONS ON VEHICULAR Technology. He was Secretary of IEEE Communication Society Finland Chapter in 1996-97 and the Chairman for years 2000-01. He has been Secretary of the Technical Program Committee (TPC) of the 2001 IEEE International Conference on Communications (ICC), and the Chair or CoChair of the Technical Program Committee of several conferences including 2006 and 2021 IEEE International Symposium on Personal, Indoor and Mobile Radio Communications (PIMRC), the Signal Processing for Communications Symposium of IEEE Globecom 2014, Symposium on Transceivers and Signal Processing for 5G Wireless and mm-Wave Systems of IEEE GlobalSIP 2016, ACM NanoCom 2018, and 2019 International Symposium on Wireless Communication Systems (ISWCS). He has also served as the General Chair of 2011 IEEE Communication Theory Workshop (CTW 2011) and 2022 IEEE Workshop on Signal Processing Advances in Wireless Communications (SPAWC).

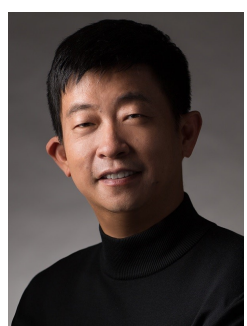

Zhu Han (S'01-M'04-SM'09-F'14) received the B.S. degree in electronic engineering from Tsinghua University, in 1997, and the M.S. and Ph.D. degrees in electrical and computer engineering from the University of Maryland, College Park, in 1999 and 2003, respectively.

From 2000 to 2002, he was an R\&D Engineer of JDSU, Germantown, Maryland. From 2003 to 2006, he was a Research Associate at the University of Maryland. From 2006 to 2008, he was an assistant professor at Boise State University, Idaho. Currently, he is a John and Rebecca Moores Professor in the Electrical and Computer Engineering Department as well as in the Computer Science Department at the University of Houston, Texas. His research interests include wireless resource allocation and management, wireless communications and networking, game theory, big data analysis, security, and smart grid. Dr. Han received an NSF Career Award in 2010, the Fred W. Ellersick Prize of the IEEE Communication Society in 2011, the EURASIP Best Paper Award for the Journal on Advances in Signal Processing in 2015, IEEE Leonard G. Abraham Prize in the field of Communications Systems (best paper award in IEEE JSAC) in 2016, and several best paper awards in IEEE conferences. Dr. Han was an IEEE Communications Society Distinguished Lecturer from 2015-2018, AAAS fellow since 2019 and ACM distinguished Member since 2019. Dr. Han is $1 \%$ highly cited researcher since 2017 according to Web of Science. Dr. Han is also the winner of 2021 IEEE Kiyo Tomiyasu Award, for outstanding early to mid-career contributions to technologies holding the promise of innovative applications, with the following citation: "for contributions to game theory and distributed management of autonomous communication networks." 


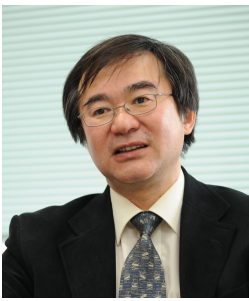

Tad Matsumoto (S'84-M'98-F'10-LF'21) received his B.S. and M.S. degrees in electrical engineering, and his Ph.D. degree in electrical engineering, all from Keio University, Yokohama, Japan, in 1978, 1980, and 1991, respectively. He joined Nippon Telegraph and Telephone Corporation (NTT), in 1980, where he was involved in a lot of research and development projects mobile wireless communications systems. In 1992, he transferred to NTT

DoCoMo, where he researched on code-division multiple-access techniques for mobile communication systems. In 1994, he transferred to NTT America, where he served as a Senior Technical Advisor of a joint project between NTT and NEXTEL Communications. In 1996, he returned to NTT DoCoMo, where he served as the Head of the Radio Signal Processing Laboratory, until 2001. He researched on adaptive signal processing, multiple-input multiple-output turbo signal detection, interference cancellation, and space-time coding techniques for broadband mobile communications. In 2002, he moved to the University of Oulu, Finland, where he served as a Professor at Centre for Wireless Communications. In 2006, he has served as a Visiting Professor with the Ilmenau University of Technology, Ilmenau, Germany, supported by the German MERCATOR Visiting Professorship Program. Since 2007, he has been serving as a Professor with the Japan Advanced Institute of Science and Technology (JAIST), Japan, while also keeping a cross-appointment position with the University of Oulu. After his retirement from JAIST, he moved to IMT-Atlantic, France, where he is serving as an invited professor. He is also Professor Emeritus of both JAIST and University of Oulu.

Prof. Matsumoto is a member of the IEICE. He has led a lot of projects supported by the Academy of Finland, European FP7, and the Japan Society for the Promotion of Science and Japanese private companies. He has been appointed as a Finland Distinguished Professor, from 2008 to 2012, supported by Finnish National Technology Agency (Tekes) and Finnish Academy, under which he preserves the rights to participate in and apply for European and Finnish National Projects. He was a recipient of IEEE VTS Outstanding Service Award, in 2001, Nokia Foundation Visiting Fellow Scholarship Award, in 2002, IEEE Japan Council Award for Distinguished Service to the Society, in 2006, the IEEE Vehicular Technology Society James R. Evans Avant Garde Award, in 2006, Thuringen State Research Award for Advanced Applied Science, in 2006, the 2007 Best Paper Award of the Institute of Electrical, Communication, and Information Engineers of Japan, in 2008, Telecom System Technology Award from the Telecommunications Advancement Foundation, in 2009, IEEE Communication Letters Exemplary Reviewer, in 2011, Nikkei Wireless Japan Award, in 2013, IEEE VTS Recognition for Outstanding Distinguished Lecturer, in 2016, and IEEE TRANSACTIONSON COMMUNICATIONS Exemplary Reviewer, in 2018. He has been serving as an IEEE Vehicular Technology Distinguished Speaker, since 2016. 